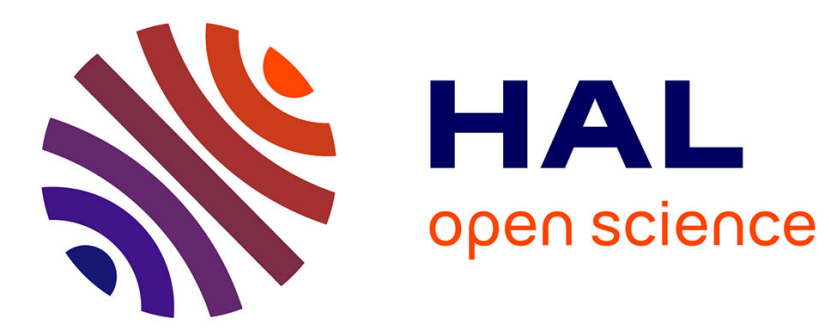

\title{
La fondation du culte du Devarāja. Danse, sacrifice et royauté au Prasat Thom de Koh Ker Éric Bourdonneau
}

\section{To cite this version:}

Éric Bourdonneau. La fondation du culte du Devarāja. Danse, sacrifice et royauté au Prasat Thom de Koh Ker. Comptes-rendus des séances de l'Académie des inscriptions et belles-lettres, 2011, 155 (3), pp.1343 - 1382. 10.3406/crai.2011.93294 . halshs-01885988

\section{HAL Id: halshs-01885988 \\ https://shs.hal.science/halshs-01885988}

Submitted on 2 Oct 2018

HAL is a multi-disciplinary open access archive for the deposit and dissemination of scientific research documents, whether they are published or not. The documents may come from teaching and research institutions in France or abroad, or from public or private research centers.
L'archive ouverte pluridisciplinaire HAL, est destinée au dépôt et à la diffusion de documents scientifiques de niveau recherche, publiés ou non, émanant des établissements d'enseignement et de recherche français ou étrangers, des laboratoires publics ou privés. 
La fondation du culte du Devarāja. Danse, sacrifice et royauté au Prasat Thom de Koh Ker

Éric Bourdonneau

Citer ce document / Cite this document :

Bourdonneau Éric. La fondation du culte du Devarāja. Danse, sacrifice et royauté au Prasat Thom de Koh Ker. In: Comptes rendus des séances de l'Académie des Inscriptions et Belles-Lettres, 155e année, N. 3, 2011. pp. 1343-1382; doi : $10.3406 /$ crai.2011.93294

http://www.persee.fr/doc/crai_0065-0536_2011_num_155_3_93294

Document généré le 19/02/2018 


\title{
COMMUNICATION
}

\author{
LA FONDATION DU CULTE DU DEVARĀJA. \\ DANSE, SACRIFICE ET ROYAUTÉ AU PRASAT THOM DE KOH KER, \\ PAR M. ÉRIC BOURDONNEAU
}

Dans la première moitié du $\mathrm{X}^{\mathrm{e}}$ siècle, Angkor-Yaśodharapura fut éclipsé, le temps d'un règne, par l'ancienne ville de Chok Gargyar, correspondant au site actuel de Koh Ker, situé $80 \mathrm{~km}$ plus au nordest (fig. 1). Il s'agit de la capitale éphémère fondée par le souverain Jayavarman IV (r. 921-941) qui abandonna Angkor où semblent avoir régné jusqu'en 928 les fils de Yaśovarman (r. 889-ca. 910). Ce déplacement de la capitale en dehors de la plaine de Siem Reap demeura un événement unique dans la période angkorienne. Si l'épisode a souvent été pris pour une simple parenthèse ou une période de bouleversements à la postérité limitée, les recherches menées ces trois dernières années suggèrent qu'il s'agit au contraire d'un moment fondateur dans l'histoire du Cambodge ancien ${ }^{1}$. C'est là, tout au moins, ce que le présent exposé souhaite illustrer à travers l'étude du grand sanctuaire royal construit par Jayavarman IV, le Prasat Thom. Sur de nombreux points, les formules du discours politico-religieux tenu jusqu'alors par les rois angkoriens, s'y trouvent profondément renouvelées. En particulier, c'est au Prasat Thom, comme nous allons le voir, qu'apparaît pour la première fois la mention de ce que la fameuse inscription de Sdok Kak Thom (K. 235) désignera plus tardivement comme le culte du Devarāja, culte rendu à la divinité tutélaire de la royauté angkorienne.

Rappelons que la nature exacte de ce culte est l'un des sujets qui a le plus fait couler d'encre dans la littérature. Depuis les travaux pionniers de Louis Finot et surtout George Cœè̀s jusqu'à ceux plus récents de Ian Mabbett, Claude Jacques ou Hiram Woodward, en y ajoutant ceux de nombreux indianistes - ainsi Adhir Chakravarti, Jean Filliozat, Hermann Kulke, Teun Goudriaan et Alexis Sanderson -, les contributions ont été nombreuses à ce qui est devenu au fil du temps l'objet d'un débat. L'une des réflexions 


\section{COMPTES RENDUS DE L'ACADÉMIE DES INSCRIPTIONS}

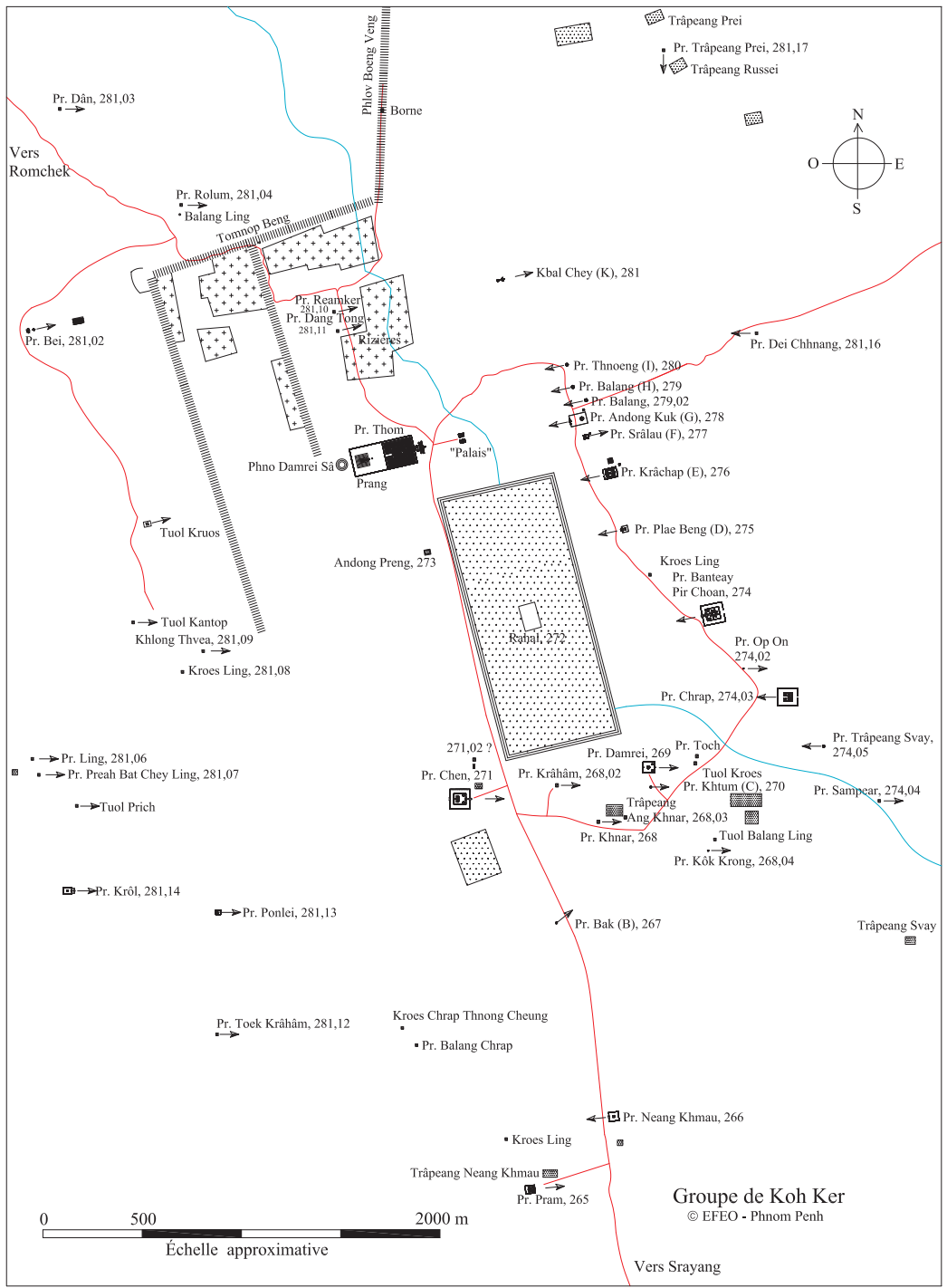

FIG. 1. - Le groupe archéologique de Koh Ker (réalisation : Chrin Narong sous la direction de B. Bruguier). 
récurrentes de l'historiographie sur la question a été de souligner la pauvreté des sources disponibles pour nous renseigner sur l'histoire de ce culte, de sorte que l'on a pu même douter de son importance véritable, après en avoir fait l'une des institutions centrales du Cambodge angkorien. À en juger par le très faible nombre d'occurrences du terme même de Devarāja dans l'épigraphie, le constat ne semble pas douteux. L'une des ambitions de la présente introduction à l'étude du Prasat Thom sera de montrer que nous ne sommes pourtant pas aussi démunis qu'il n'y paraît, dès lors que l'attention ne se focalise plus sur la seule stèle de Sdok Kak Thom (et le terme de devarāja) et dès lors que l'analyse des inscriptions s'élargit à celle des dispositifs iconographiques et architecturaux. La thèse défendue est que les unes ne vont pas sans les autres et qu'on ne saurait rouvrir le dossier sur le Devarāja sans considérer ce que les images et les monuments nous disent sur la scène rituelle où celui-ci apparaît pour la première fois.

Il s'agit ce faisant de redonner à Koh Ker la place qui lui revient dans l'histoire de ce culte. Force est de constater que, mis à part les travaux de George Cœdès, l'historiographie ne s'y est guère attardée. Les raisons en sont diverses, et nous pouvons d'ores et déjà mentionner parmi celles-ci la position excentrée du site. La destinée de Koh Ker en aura ainsi pâti à deux moments décisifs de son histoire : au milieu du $\mathrm{X}^{\mathrm{e}}$ siècle tout $\mathrm{d}$ 'abord, lors de son abandon comme capitale, avec le retour du centre du pouvoir dans la plaine de Siem Reap sous le règne de Rājendravarman (r. 944-967/968) ; lors de sa redécouverte ensuite, au XIX ${ }^{\mathrm{e}}$ et au XX $\mathrm{XX}^{\mathrm{e}}$ siècle, les travauX pionniers de Henri Parmentier ${ }^{2}$ n'ayant guère suffi à détourner l'attention des architectes et des archéologues du site d'Angkor - si bien que, jusqu'à ces dernières années, les temples de Koh Ker sont demeurés vierges de toute nouvelle mission archéologique pour devenir une cible privilégiée des pilleurs ${ }^{3}$.

\section{Le Prasat Thom, demeure de Śiva Tribhuvaneśvara}

Un petit groupe d'une dizaine d'inscriptions est gravé sur les murs et les piliers du Prasat Thom. Deux d'entre-elles livrent ce qui se présente comme la date de fondation du temple. L'une est en

2. Parmentier 1939, chap. I « Le groupe de Koh Ker », p. 15-117.

3. Bourdonneau 2011. 
sanskrit et accompagne la mention de la date de précisions astrologiques permettant d'identifier le jour et l'heure approximative de celle-ci : le mercredi 12 décembre 921 , « vers 8 h47 ${ }^{4}$. Il s'agit du $10^{\mathrm{e}}$ jour de la quinzaine claire du mois de Pauṣa, en l'année 843 de l'ère śaka, 10 jour désigné comme le « jour de Yama », ce qui n'est peut-être pas indifférent dans le cas présent, comme nous allons le voir.

Nous reproduisons ci-dessous la traduction proposée par G. Cœdès des premières stances de cette inscription, inscription en assez mauvais état de conservation dont Cœdès a certainement tiré le maximum de ce qui pouvait être lu :

Fortune! Succès! Bonheur! Victoire !

I. Celui qui, sans commencement lui-même, est le commencement (le principe) du monde entier, à commencer par le dieu aux quatre visages, qui ... étant sans corps, prend cependant huit corps....... dans la demeure nommée Tribhuvaneśvara.

II. Le roi des Śakas est (figuré) par les (trois) feux, les (quatre) mers et les (huit) Vasus ; le jour de Yama, dans la quinzaine claire de Paușa ; le soleil avec Mercure (est dans le ...) ; Vénus et l'horoscope sont ... dans le Verseau; Saturne est dans la Balance ; tels sont, dans l'ordre, les temps propices...

III. À ce Tri[bhuvaneśvara] qui l'a fait maître de tous les maîtres de la terre, en même temps qu'un moyen de ... le succès ........... ce [Śrī Jaya]varman victorieux a donné avec dévotion tout ceci, merveille et essence de la royauté [ou du royaume $]^{5}$.

Nous y apprenons ainsi que le temple était dédié à Śiva Tribhuvaneśvara, «Seigneur des trois mondes ». C'est bien ce vocable dont les inscriptions plus tardives ont conservé le souvenir lorsqu'elles évoquent le règne de Jayavarman IV, célébré comme le «fondateur du siège de Tribhuvana ».

L'ensemble des autres inscriptions du Prasat Thom remonte également au règne de Jayavarman IV (921-941) sauf une (K. 682, 11. 19-46), portant la date 923 ś/1001 A.D, correspondant au règne éphémère de Udayādityavarman I. Le temple était donc encore en activité à cette époque. La vie du Prasat Thom et de Koh Ker de manière plus générale ne s'est donc pas arrêtée avec la mort de Jayavarman IV, mais il demeure que la réalisation du Prasat Thom

4. K. 184, Billard 2006, p. 406.

5. Cf. Cœdès 1931 , p. 13 et la correction apportée dans Cœdès 1952, p. 257. Stance III, la traduction de Cœdès restitue «Tribhuvaneśvara " mais l'auteur propose en réalité de lire « Tribhuvaneśa », restitution davantage respectueuse de la métrique (Cœedès 1931, p. 13, n. 3). 
doit toute entière être attribuée au règne de ce roi. Certes, la chronologie interne à sa période de construction a pu et a été relativement complexe et, à l'évidence, le temple est resté inachevé. Le Prasat Thom n'en montre pas moins une remarquable cohérence, à la fois architecturale et iconographique, comme nous allons le voir à présent.

Le sanctuaire se subdivise assez clairement en trois pôles, alignés sur un même axe sud-ouest/nord-est (fig. 2).

Un premier pôle est formé d'un dispositif à la fois concentrique et axé : une douve et trois enceintes, emboîtées les unes dans les autres sont traversées par une chaussée d'accès, elle-même jalonnée de quatre pavillons d'entrée et s'étirant considérablement vers l'est pour conduire, semble-t-il, à un vaste bassin aujourd'hui à sec.

Nous ne rentrerons pas dans le détail de ce premier dispositif et nous nous contenterons de quelques remarques. Les édifices de la première enceinte retiendront tout d'abord notre attention : la tour centrale, précédée d'un maṇdapa (pavillon axial), y est entourée de huit autres tours alignées en deux rangées. G. Cœdès a proposé de localiser dans chacune de ces huit tours les huit mūrti ou manifestations de Śiva, telles qu'elles sont évoquées dans l'inscription sanskrite : Śiva « étant sans corps, prend cependant huit corps » (Cœdès 1965, p. 63). La tour principale, celle du centre de la rangée orientale, abritait selon toute vraisemblance l'image de Śiva Tribhuvaneśvara sous la forme d'un linga.

Ces tours présentent des dimensions très réduites et, de façon remarquable, la tour principale est encore sensiblement plus petite que les tours placées dans la seconde rangée. La porte de la cella dépasse ainsi difficilement le mètre de hauteur. Cette différence de proportion est là l'application d'un principe qui détermine l'ensemble de la composition du sanctuaire : l'agrandissement progressif, du centre vers la périphérie, des dimensions des édifices et, dans une certaine mesure, de la taille des images (nous y reviendrons).

Ce principe s'illustre de façon la plus spectaculaire dans la série des quatre pavillons d'entrée orientaux. Les gopura III et IV atteignent ainsi des dimensions colossales, l'un en élévation, l'autre en plan. Ils offrent là l'image parlante de " l'expansion cosmique du temple », comme cela a pu être évoqué à propos des temples plus tardifs du pays tamoul, expansion « propagée d'enceinte en enceinte, de plus en plus loin et de plus en plus haut » (Pichard 1994, p. 20). 


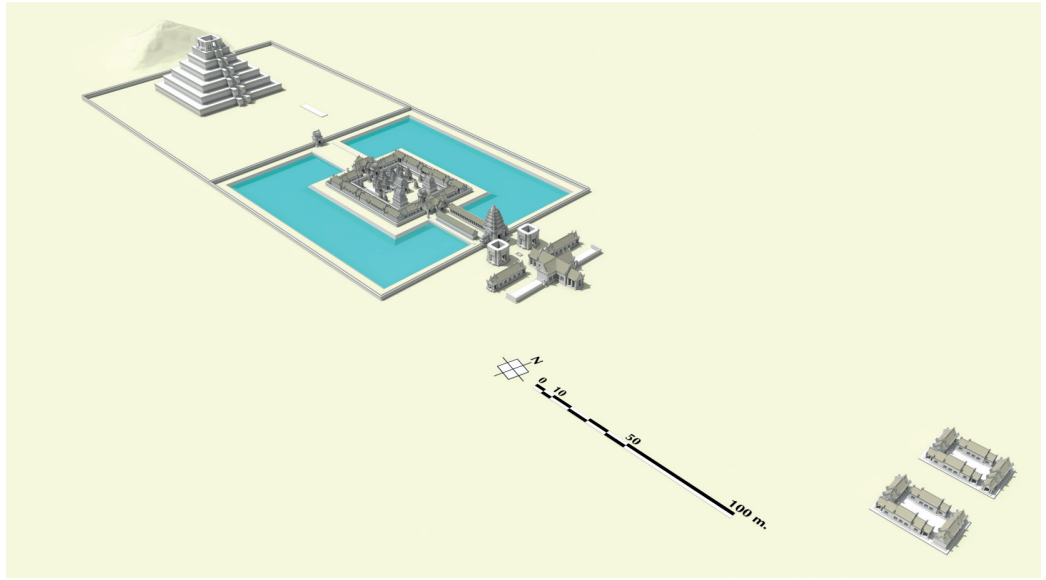

FIG. 2. - Restitution archéologique tridimensionnelle du Prasat Thom (travail en cours de O. Cunin).

Un temple-montagne aux dimensions et aux proportions impressionnantes forme le second pôle du complexe rituel du Prasat Thom. Il s'agit d'une pyramide en grès composée de cinq gradins massifs, s'élevant d'un seul jet à $36 \mathrm{~m}$ de hauteur.

Cette pyramide, flanquée d'un seul escalier, sur sa face orientale, est entourée d'une enceinte percée à l'est d'une unique porte. Son implantation s'inscrit ainsi rigoureusement dans le plan axé du Prasat Thom. L'enceinte de la pyramide vient s'appuyer sur celle entourant la douve. Il est ainsi fortement probable que la consécration de la pyramide ait été postérieure de quelques années à la date de 921, hypothèse en accord avec le temps nécessaire à l'élévation d'une telle masse de pierre.

Au sommet de la pyramide, une tour inachevée abrite les vestiges d'un piédestal réalisé à l'échelle d'un véritable édifice et dont le poids de chacun des blocs devait dépasser les dix tonnes. Il donne une première idée des dimensions elles-mêmes hors norme du linga qu'il enveloppait.

Trois inscriptions concordantes, dont deux retrouvées à Koh Ker, célèbrent l'exploit d'une telle réalisation. L'une d'entre elles, gravée au Prasat Damrei, le rapporte en ces termes :

« Lui [Jayavarman IV] qui, charmant l'esprit des gens d'esprit, pulvérisant l'orgueil des orgueilleux établit un linga d'Ugra de neuf [parts], très lourd, consistant en neuf coudées » (trad. Filliozat 1981, p. 77). 
Si nous restituons une coudée à $45 \mathrm{~cm}$, nous obtenons un linga qui aurait mesuré environ $4 \mathrm{~m}$ de haut.

À l'extrémité ouest du complexe du Prasat Thom, quatre petits temples (peut-être cinq à époque ancienne) abritent des ling $a$ atteignant des dimensions également imposantes, faisant ainsi écho au linga de la pyramide. Ces quatre temples sont implantés sur un axe qui fait face au Prasat Thom et font à l'évidence partie du même dispositif. Nous avons ainsi une composition grandiose qui s'étire d'est en ouest sur plus de $1 \mathrm{~km}$.

Il faut se rendre sur le site pour prendre la mesure de ce contraste saisissant entre d'une part une pyramide massive de $35 \mathrm{~m}$ de haut et, d'autre part, la minuscule tour en brique placée au cœur du sanctuaire. Une curieuse hypothèse aura été de supposer que cette toursanctuaire en réduction avait pu être bâtie comme un temple provisoire en attendant l'achèvement de la pyramide (Parmentier 1939, p. 114).

Dans ce dédoublement sur un même axe du saint des saints et du linga, il semble au contraire que l'on doive reconnaître la juxtaposition de deux conceptions du divin. Nulle part ailleurs au Cambodge, le jeu sur les proportions, où l'infiniment petit côtoie l'infiniment grand, n'est peut-être aussi signifiant : là où la pyramide à gradins, conçue comme le gigantesque piédestal d'un linga démesuré, proclame aux yeux de tous la gloire et la souveraineté du dieu, en même temps que celle du roi à l'origine d'un tel exploit, la conception sous-jacente à la cella en brique, construction en apparence, et par comparaison, bien dérisoire, met l'accent sur l'inaccessibilité de la divinité au «corps subtil comme un grain de sésame », pour reprendre ici une formule rencontrée dans l'épigraphie ${ }^{6}$.

Il faut ici avoir à l'esprit les grandes conceptions théologiques qui traversent très largement le śivaïsme du Cambodge ancien. À son plus haut degré de transcendance, Śiva, divinité suprême, demeure au-delà des capacités humaines de conception et de perception; ce n'est qu'au long d'une chaine d'émanations hiérarchisées, par ordre de subtilité décroissante, que le dieu se manifeste et s'offre au culte.

Je renvoie brièvement à ce sujet, entre autres travaux, à ceux de M. Pierre-Sylvain Filliozat qui a exposé ici-même quelques-uns des principes fondamentaux qui président à ce mouvement émanateur de la divinité, tel qu'il a été mis en forme, selon l'auteur, dans les

6. K. 1155, st. I (trad. Gerschheimer et Estève dans Estève 2009, p. 535). 
temples du Karnātaka, en accord avec les écrits du śivaïsme siddhānta (Filliozat 2001).

Au Prasat Thom de Koh Ker, entourée de ses trois enceintes concentriques, masquée derrière des gopura successifs, précédée d'un mandapa, la petite tour qui abrite le "Seigneur des trois mondes » tout en dérobant celui-ci au regard du fidèle, met précisément en scène cette inaccessibilité du dieu. Elle est placée également au départ de ce mouvement d'ouverture au monde que nous venons d'évoquer, repoussant à la périphérie la pleine manifestation de l'essence divine : en l'occurrence ici, dans le gopura monumental de la troisième enceinte, abritant comme nous allons le voir, l'une des statues les plus remarquables du Cambodge angkorien.

Tout en retravaillant un vocabulaire antérieur - on se tournera là, en priorité, vers les temples du groupe de Roluos, mais sans omettre de se référer aux réalisations, plus anciennes, de Sambor Prei Kuk -, les maîtres d'œuvre du Prasat Thom ont défini une formule architecturale inédite appelée à une postérité considérable.

Un plan axé s'étirant en longueur, l'agrandissement progressif, vers la périphérie, des dimensions des pavillons d'entrée, l'adoption d'un mandapa devant la tour centrale, sont en effet autant de caractéristiques qui, entre autres éléments distinctifs, vont se retrouver, dans les décennies et les deux siècles qui suivent, à Banteay Srei, au Phnom Chisor, à Preah Vihear ou encore à Vat Phu, pour ne citer ici que des sites de temple parmi les plus célèbres, mais il en existe de nombreux autres. Cette formule architecturale, qui va de pair avec un programme iconographique et une titulature divine qui se fixent aussi à Koh Ker, est fondamentalement celle de sanctuaires de 《 lieux saints ». Elle met en scène la présence divine extraordinaire qui y est reconnue, identifiée généralement à un élément ou une portion même du paysage, c'est-à-dire perçue comme s'étant manifestée ici-bas en dehors de toute détermination humaine.

Ces lieux saints font, pour certains d'entre eux, l'objet de pèlerinages (aristocratiques et royaux) bien attestés dans l'épigraphie. De ce point de vue, la lecture proposée précédemment de ce plan axé demeure évidemment incomplète. Au mouvement émanateur de la divinité, répond celui inverse de la résorption cosmique dans le divin qui est également celui du fidèle faisant retour à la source divine (Padoux 2010, p. 86). Le dispositif adopté nous apparaît là comme la mise en œuvre originale de cet effet de "magnitudes inversées » évoqué par Peter Brown à propos de sanctuaires de lieux saints dans 
un contexte naturellement bien différent. L'auteur entendait caractériser par cette formule l'effet selon lequel « l'efficacité surnaturelle est d'autant plus forte que l'acte ou l'objet religieux est plus "dense" » : ainsi la pierre brute recélant à l'état « concentré » la pleine puissance du dieu (ou du saint), ainsi les derniers pas du dévot au terme de son pèlerinage ressentis comme les plus décisifs? L'extension de la chaussée d'accès et le jalonnement des gopura successifs maintiennent soigneusement une tension entre distance et proximité - le dévot parvenu au plus près du divin, celui-ci paraît se dérober à chaque franchissement d'un nouveau pavillon d'entrée. Il en résulte un sens aiguisé de la présence divine, à la fois inaccessible et éminemment proche. L'enjeu demeure naturellement de comprendre les raisons pour lesquelles une telle proximité de l'inaccessible trouve précisément à s'illustrer à partir de la première moitié du $\mathrm{X}^{\mathrm{e}}$ siècle. Une telle compréhension exige, on s'en doute, de prendre la mesure des évolutions globales de la société angkorienne. Disons très brièvement qu'il y a là une manière inédite de mettre en scène l'établissement du divin dans le monde - à laquelle contribue le développement de l'iconographie et le renouvellement de la titulature et de l'onomastique divines (cf. infra) -, au moment même où s'affirme ce que nous avons provisoirement proposé de désigner, en se référant à une expression bien attestée dans les inscriptions, comme un processus d'affermissement de la « surface d'en-bas »(phdai karom $)^{8}$.

Le troisième pôle que l'on peut identifier au sein du complexe formé par le Prasat Thom est une étonnante colline artificielle de $22 \mathrm{~m}$ de haut, située immédiatement à l'ouest de la pyramide du Prasat Thom, et désignée aujourd'hui comme la « tombe de l'éléphant blanc » (phno damrei sâ). Elle demeure aujourd'hui un lieu de culte auquel est attaché un récit légendaire très populaire au Cambodge et qui donne son nom à la colline.

Nous y avons mené une première campagne de fouilles en 2009. Elle a permis d'établir que la colline était bien sensiblement contemporaine du Prasat Thom et du temple-montagne en particulier. Les fouilles ont par ailleurs révélé, à l'intérieur de la colline, la présence d'une couche sableuse mêlée de cendres et extrêmement riche en charbons de bois. Une seconde campagne, réalisée en 2010, eut pour

7. Brown 2007 [1984], p. 114-115 et Schmitt 1984, p. 289.

8. Bourdonneau 2011, p. 99-100. 
objectif de documenter la structure de la colline sur l'ensemble de sa profondeur, en réalisant une série de carottages. Ceux-ci ont ainsi permis de montrer que la couche cendreuse documentée en 2009 était d'une profondeur considérable, avoisinant les $16 \mathrm{~m}$ d'épaisseur et s'étendant d'est en ouest sur plus de $40 \mathrm{~m}$ de long. L'origine d'une telle quantité de charbons de bois, et le souci de les ramener ainsi au cœur de la colline, soulèvent naturellement de nombreuses interrogations. Nous ne nous y attarderons pas ici mais il va de soi que les réponses qui pourront y être apportées seront en mesure d'éclairer de façon décisive le complexe rituel du Prasat Thom. Malheureusement, la masse d'une telle structure, d'une part, son caractère sacré, d'autre part, ne permettent pas pour l'heure de poursuivre les interventions sur le terrain. Nous ne sommes pas totalement démunis néanmoins pour intégrer ces premières observations dans une interprétation globale du Prasat Thom. Les pratiques rituelles du Cambodge plus récent offrent, en effet, certaines pistes de réflexion; c'est le cas, en particulier, des anciennes cérémonies dites du « troisième mois » (mois de mãgh), telles qu'elles ont été décrites notamment par Adhémard Leclère, cérémonies qui associent « fête de l'offrande du feu » et « entassement des monts de paddy » ou « des monts de sable ${ }^{9}$.

\section{Retour sur le Devarāja}

Avant de se tourner vers le programme iconographique, il importe de considérer à nouveau l'épigraphie du monument, en s'attachant cette fois-ci aux inscriptions en vieux khmer. Elles sont les plus nombreuses. L'expression par laquelle elles désignent le Śiva Tribhuvaneśvara de l'inscription sanskrite retient d'emblée l'attention. Il s'agit de la formule vrah kamraten añ jagat ta rājya (le pronom añ est omis dans un cas, le terme jagat dans un autre). Elle se retrouve dans les inscriptions plus tardives de l'époque angkorienne et, en particulier, dans la plus célèbre d'entre elles, la stèle de Skok Kak Thom, où apparaît un nouveau composé sanskrit, sensiblement équivalent : devarāja. Dans l'historiographie, c'est davantage ce dernier terme qui a été placé au cœur des vives discussions menées sur l'histoire de ce culte - discussions qui, nous l'avons dit, n'ont guère accordé au Prasat Thom de Koh Ker et, partant, aux

9. Leclère 1917 , p. 292-320. 
inscriptions en vieux khmer de ce monument toute la place qui leur revient. Si la position excentrée du site et le nombre réduit des travaux qui lui ont été consacrés expliquent en partie une telle lacune, d'autres raisons intéressent plus directement l'analyse des inscriptions et nécessitent que l'on s'y arrête un peu plus longuement.

L'une d'entre elles tient à la mise en cause de l'identification proposée très tôt par G. Cœdès entre les deux expressions : tribhuvaneśvara d'une part, vrah kamraten añ jagat ta rājya de l'autre. Partant du constat que l'une et l'autre n'apparaissaient ensemble dans aucune inscription, il a été supposé que les deux expressions désignaient des divinités non seulement distinctes mais appartenant à des sphères radicalement différentes. Les inscriptions khmères s'adresseraient à une sorte de génie du territoire, génie chargé de veiller à la seule prospérité d'un royaume supposé de Koh Ker, qui ne serait pas forcément représenté matériellement, ni abrité par aucun des édifices du Prasat Thom : elles désigneraient une réalité proprement khmère et locale, opposée à une réalité qui serait proprement indienne et qui est identifiée avec le Śiva Tribhuvaneśvara du texte sanskrit (Jacques 2009, p. 17). Rien du dispositif cultuel du Prasat Thom ne serait ainsi en mesure de nous renseigner sur le culte du devarāja.

Les objections à une telle distinction de deux niveaux de « réalité » sont nombreuses et elles sont décisives. Nous n'en mentionnerons ici que quelques-unes, en commençant tout d'abord par relever la localisation des inscriptions au sein même du sanctuaire (fig. 3).

L'inscription sanskrite (K. 184) mentionnant la donation somptueuse faite en 921 à Tribhuvaneśvara par le roi Jayavarman IV est gravée à l'intérieur du gopura est de la première enceinte. La plus ancienne inscription en vieux khmer mentionnant le vrah kamraten añ jagat ta rājya (K. 682) apparaît, elle, à quelques pas de là, sur l'un des piliers du porche ouest du second gopura oriental. Elle est, en d'autres termes, gravée aussi près que possible de l'inscription précédente, en suivant la relation hiérarchique établie entre les édifices. L'objet de cette inscription est la mention d'une donation faite la même année, en 921, par un nommé Cikhāvindu, désigné comme khloñ vnam, c'est-à-dire comme le « chef du sanctuaire » (cette dernière expression est bien attestée dans l'épigraphie et ne souffre d'aucune ambiguïté : vnam est ici le temple où est gravée l'inscription). La juxtaposition des inscriptions dans l'espace du 


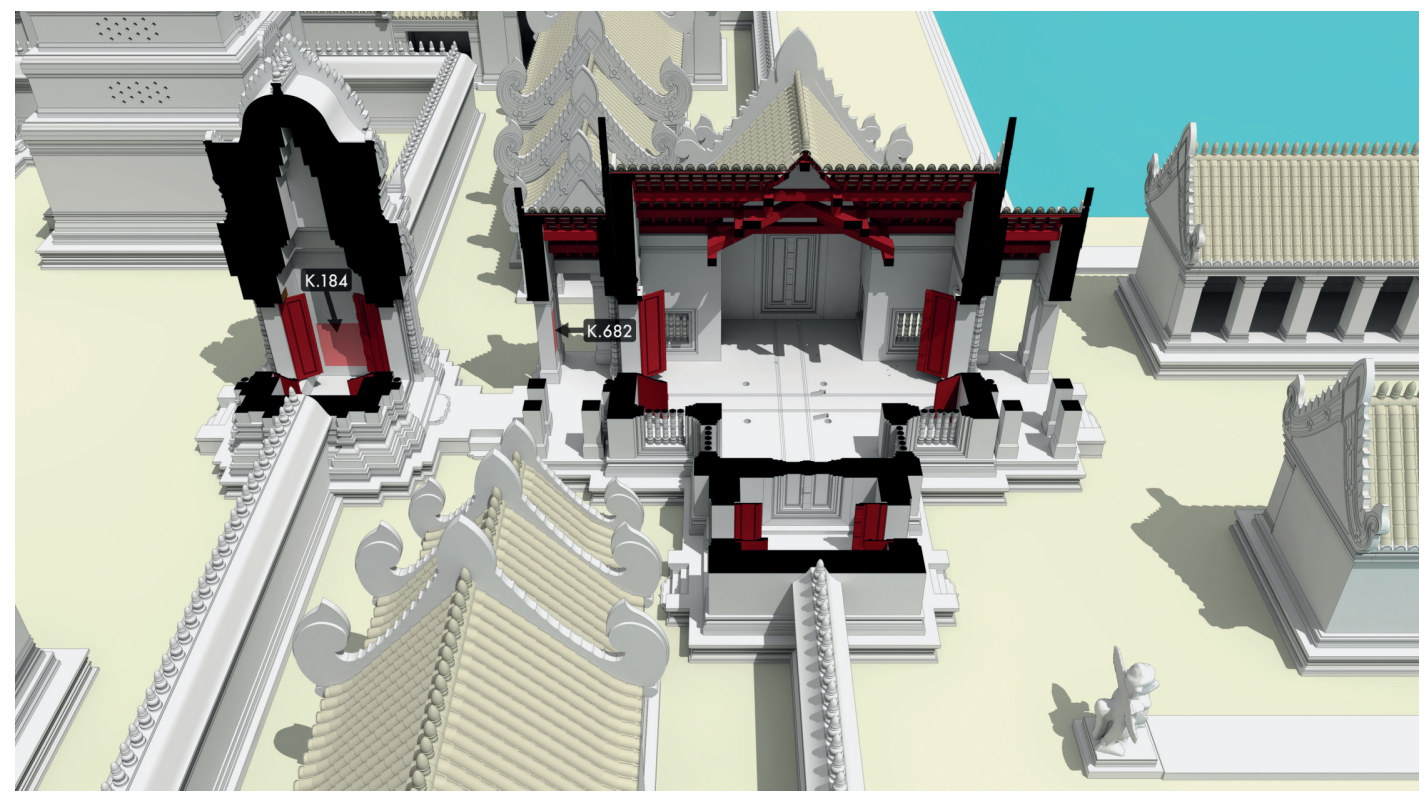

FIG. 3. - Partie centrale du Prasat Thom, localisation des inscriptions K. 184 et K. 682 (restitution archéologique tridimensionnelle : travail en cours de O. Cunin).

sanctuaire n'offre ainsi rien que de très familier et un tel dispositif ne s'écarte en aucune façon de ce que montrent par ailleurs massivement les centaines de temples qui nous sont connus (où l'on retrouve ce même ordre dans l'exposé des œuvres royales et de celles des dignitaires, cette même hiérarchie entre le sanskrit et le vieux khmer).

L'examen des deux expressions en elles-mêmes s'oppose tout autant à la thèse de « deux niveaux de réalité ». La compréhension du composé sanskrit tribhuvaneśvara ne se prête évidemment pas à contestations. Il se décompose en trois parties : î́vara « seigneur », bhuvana «monde» et tri- pour «trois». Rappelons à nouveau qu'à ce "Seigneur des trois mondes », Jayavarman IV, devenu le « maître de tous les maîtres de la terre » donne avec dévotion sārādbhutam räjyasya « la merveille de son royaume (rājya) et ce qui se trouve le plus précieux».

L'expression vrah kamraten añ jagat ta räjya peut être décomposée comme suit. Les trois premiers termes vrah kamraten a forment la titulature divine usuelle de l'époque. Le terme $v \mathrm{rah}$ peut 
être défini comme une «particule honorifique »; kamraten est généralement traduit par «maître ou seigneur»; enfin, añ est interprété comme un «pronom de la première personne » (Cœedès 1961). La particule ta permet d'établir un rapport de détermination. Le terme jagat est un mot emprunté au sanskrit et peut être traduit par «monde» ou « univers ». Rājya, nous l'avons vu, désigne le « royaume» $($ ou la « royauté »).

Nous avons ainsi, d'une part, un «Seigneur des trois mondes» qui reçoit en offrande le plus précieux ou l'essence du « royaume » et, d'autre part, ce qui peut être compris comme un «Seigneur de l'univers ou des mondes» qui est également Seigneur «du royaume ». L'équivalence n'est, à tout le moins, pas si mauvaise ${ }^{10}$.

L'examen des inscriptions plus tardives où réapparaît l'expression khmère forgée au Prasat Thom, est tout aussi instructif. En dehors du site de Koh Ker, nous en comptons aujourd'hui six qui s'échelonnent entre le règne de Jayavarman V (r. 968-1000/1001) et celui de Jayavarman VII (r. 1180-env. 1218/1220) ${ }^{11}$.

Ces inscriptions nous permettent de suivre avec une certaine précision l'évolution et la transformation progressive de l'expression au fil des décennies. Nous rencontrons à la fin du $\mathrm{X}^{\mathrm{e}}$ siècle la forme abrégée rencontrée ponctuellement à Koh Ker-vrah kamratè añ ta rājya - puis, au début du $\mathrm{XI}^{\mathrm{e}}$ siècle, vrah kamraten் añ ta rāja (avec la substitution de rāja, le « roi », à rājya); au milieu de ce même XI ${ }^{\mathrm{e}}$ siècle, dans la stèle de Skok Kak Thom, nous retrouvons très ponctuellement cette dernière forme mais c'est l'expression kamraten jagat ta rāja qui s'impose désormais (rāja étant toujours substitué à rājya) et qui réapparaitra un siècle et demi plus tard au Bayon. Nous avons vu que cette stèle livre désormais, dans la partie sanskrite de l'inscription, un nouvel équivalent de cette expression, le composé devarāja, dans lequel deva est supposé valoir pour kamraten jagat.

10. À l'appui d'une telle équivalence, il importe de mentionner également une inscription provenant du Phimeanakas (au cœur du palais royal à Angkor), de peu antérieure au corpus du Prasat Thom et où, à notre connaissance, apparaît pour la première fois la formule kamraten jagat. Or celle-ci semble désigner ici un Viṣnu Trailokyanātha, c'est-à-dire précisément Viṣṇu « Seigneur ou protecteur des trois mondes » (K. 291, piédroit sud st. X, piédroit nord 1. 1-2 et 1. 26, cf. Coedès 1951, p. 201-202).

11. Une septième inscription, également postérieure au règne de Jayavarman IV, vient s'y ajouter : l'inscription du Prasat Thom déjà citée, K. 682, qui reproduit, pour sa part, fidèlement la formule vrah kamraten añ jagat ta rājya des inscriptions plus anciennes de Koh Ker. 
Parmi ce petit groupe d'inscriptions, dont les analyses demanderaient à être reprises une à une, nous retiendrons tout d'abord un texte daté du règne de Jayavarman $\mathrm{V}$, qui n'est publié à ce jour que de façon incomplète et qui s'oppose assez nettement à la thèse de " deux niveaux de réalité ». Il relate en effet à deux reprises, tout d'abord dans la partie sanskrite (récemment étudiée par Gerdi Gerschheimer) puis dans le texte khmer, l'accomplissement d'un même rite "d'ouverture des yeux » (unmillita) d'une divinité désignée dans le premier comme vrah kamraten añ ta rājya et dans le second comme Śiva Maheśvara sous la forme du ling $a^{12}$.

Il nous faut bien sûr revenir également sur le contenu de l'inscription de Skok Kak Thom. Si celle-ci constitue sans nul doute une source majeure pour l'histoire du Devarāja, l'usage qui en a été fait dans la littérature explique aussi pour beaucoup le fait que le Prasat Thom de Koh Ker n'ait guère reçu l'attention qu'il méritait.

L'inscription rapporte qu'en 802, le roi Jayavarman II invita un brahmane nommé Hiranyadāma, versé dans la science des Mantras source de siddhi (c'est-à-dire de succès mais aussi de pouvoirs surnaturels), à « accomplir une cérémonie telle qu'elle rendît impossible pour ce pays-ci des Kambuja toute allégeance vis-à-vis de Javā, telle qu'elle rendît possible l'existence d'un maître sur terre (kamraten phdai karom, litt. "seigneur de la surface d'en-bas") absolument unique, qui fût cakravartin ("souverain universel") ». Ce brahmane, poursuit l'inscription, fit un rituel selon le vénérable Vināśikha et installa le kamraten jagat ta rāja. Puis il récita «le saint Viṇāsikha, le Nayottara, le Sammoha et le Śiraścheda (...) pour les enseigner au sten añ Śivakaivalya » (ce dernier étant le lointain ancêtre de l'auteur de l'inscription, Sadāśiva) ${ }^{13}$.

Deux stances de la partie sanskrite de l'inscription précisent pour leur part :

St. XXVIII. Les śastras appelés Śiraścheda (litt. la « décollation»), Vināsikha, Sammmoha, Nayottara, ces quatre faces de Tumburu, ce brahmane les lui révéla comme par le moyen de ce Siddhi.

St. XXIX. Pour l'accroissement de la prospérité du monde, ce brahmane a extrait soigneusement l'essence de (ces) śāstras par sa maîtrise des (enseignements) ésotériques et a établi (avec cette essence) les Siddhi qui portent le nom de Devarāja ${ }^{14}$.

12. K. 1141, voir Pou 2001, p. 115 (pour le texte khmer) et Estève 2009, p. 536 (édition du texte sanskrit par Gerdi Gerschheimer).

13. K. 235-C, 11. 71-75, trad. Cœdès et Dupont 1943-46, p. 109-110.

14. Trad. d'après Sanderson 2003-2004, p. 356. 
Les quatre textes mentionnés sont les sources majeures de la division dite du Vāmasrotas au sein de la tradition śivaïte non saiddhāntika (dite Vidyāpītha) du Mantramārga. Rappelons brièvement qu'il existe plusieurs classifications, assez complexes, des textes śivaïtes. L'une d'entre elles répartit les tantra śivaïtes entre Atimārga, une " voie extérieure », plus ancienne et réservée aux ascètes cherchant la libération, et Mantramārga, une "voie des mantras », où l'on recherche aussi bien les pouvoirs surnaturels que la libération (Padoux 2010, p. 61). Selon A. Sanderson, le courant Vāma du Mantramārga a pu occuper dans la seconde moitié du premier millénaire une position prééminente au sein du śivaïsme avant d'être éclipsé par les autres traditions du Vidyāpīṭa (Sanderson 2009, p. 129, n. 301). Ce courant était centré sur le culte de Śiva-Tumburu, ainsi qu'il est mentionné dans l'inscription, accompagné d'un petit groupe de déesses identifiées comme les sœurs de Śiva.

Nous devons à Teun Goudriaan d'avoir proposé une première étude rassemblant les sources nous renseignant sur la figure de Tumburu, ainsi qu'une édition du Vīnāśikha, tel que celui-ci a survécu dans un unique manuscrit de feuille de palme conservé au Népal. Tumburu y apparaît sans surprise comme une divinité offrant une dimension royale, mais aussi comme un dieu guérisseur, prolongeant la vie, une divinité protectrice mais ambivalente car capable aussi d'exercer des pouvoirs destructifs. Cette ambivalence est concrétisée par les terribles déesses qui l'accompagnent et l'entourent. Elles sont au nombre de quatre ou davantage. Dans le premier cas, comme dans le Viṇāsikha, les quatre sœurs de Śiva portent des noms allégoriques, formant autant d'expressions des différents aspects de la victoire (Jayā, Vijayā, Jayantī/Ajitā et Aparājitā); dans le second, elles sont apparentées à l'occasion à la série des Mātrkā, les sept ou huit " Mères ». Tumburu se tient à leur tête et peut s'opposer à leur influence néfaste (Goudriaan 1985, p. 23).

L'ensemble de ces textes décrivent Tumburu comme étant à quatre faces mais l'un d'entre eux (le Netratantra) mentionne une forme à cinq faces, dans laquelle on peut reconnaître une contamination de Sadāśiva, la divinité principale du śaivasiddhānta (le même ouvrage précise ainsi que Tumburu a un corps semblable à Sadāśiva). La relation ou la confusion (iconographique) de Tumburu avec une autre hypostase de Śiva, Vīrabhadra ou Vīreśvara, est également à relever. Une telle confusion apparaît dans les chapitres de l'Agnipurāna. Les deux figures ont notamment en commun de porter la vinnă, c'est-à-dire la cithare, d'être accompagnées d'un taureau et 
d'être associées au groupe des Mātrkā, les sept ou huit « Mères ». Marie-Thérèse Mallmann a suggéré que l'Agnipurāna puisse illustrer une étape de la substitution progressive de Vīrabhadra à Tumburu (Mallmann 1963, p. 63-65).

Il faut enfin dire quelques mots du Vinnāśikha (dont on peut tout d'abord relever que le titre de l'ouvrage présuppose un symbolisme spécifique de la vinna $)$. Si les mentions de Tumburu dans la littérature ne sont pas rares, le Viṇāsikha, note Goudriaan, est le seul texte connu qui soit exclusivement consacré au culte de celui-ci (Goudriaan, 1985, p. 18). Son importance comme l'une des sources majeures de la tradition Vāma est ainsi bien attestée dans le corpus des textes śivaïtes. Le texte lui-même se présente sous la forme d'un dialogue entre Śiva et ses parèdres, assis sur le mont Kailāsa et entourés par les gana (les troupes de Śiva) menés par la figure de Mahākala. L'une des principales caractéristiques de l'ouvrage est d'être fondamentalement préoccupée de pratiques magiques pour l'obtention de pouvoirs surnaturels directement applicables dans ce monde-ci. Exemple parmi d'autres, le culte rendu aux divinités du Vīnāśikha, le $9^{\mathrm{e}}$ jour du mois, doit permettre à un souverain d'obtenir la victoire sur ses ennemis (vs. 17). Telle autre cérémonie de huit mille libations, après avoir mélangé de la chair humaine avec du beurre, du miel et du lait, permettra d'anéantir l'ennemi de façon immédiate (vs. 162).

De cette rapide évocation des textes cités par la stèle de Sdok Kak Thom, nous pouvons tout d'abord retenir que celle-ci permet de réaffirmer avec force la dimension śivaïte du culte adressé au kamrateñ jagat ta rāja. En cela, elle s'accorde pleinement avec les données des inscriptions plus anciennes, dont celles de Koh Ker. Bien plus délicat est toutefois de déterminer si la tradition spécifique du śivaïsme tantrique à laquelle nous sommes là renvoyés (le culte de Śiva sous la forme de Tumburu) est, d'une quelconque façon, susceptible de nous renseigner sur le dispositif cultuel du Prasat Thom. Nous allons y revenir, mais convenons dès à présent que, s'ils ne sont peut-être pas décisifs, les obstacles sont nombreux.

Parmi ceux-là, il y a d'abord ce qui, dans la stèle de Sdok Kak Thom, ne s'accorde guère avec les inscriptions antérieures. Car si l'identité śivaïte du kamraten jagat ta rāja/Devarāja n'est pas contredite, les choses sont très différentes dès lors que l'on considère la date de naissance et, de manière plus générale, la chronologie de ce culte, supposée longue de 250 ans, telle qu'elle est rapportée par Sadāśiva dans la stèle de Sdok Kak Thom. Les divergences avec 
les sources plus anciennes surgissent alors quasiment à chaque étape du récit proposé.

Ainsi le récit de Sadāśiva (qui précise que seule sa lignée fut autorisée à officier devant le Devarāja) est-il, par exemple, difficilement compatible avec l'inscription du Prasat Thom, datée de l'an 1001, attestant l'activité du culte à Koh Ker sous le règne de Udayādityavarman $\mathrm{I}^{\mathrm{er}}$. Elle s'accorde également assez peu avec les témoignages de l'existence de cultes provinciaux du vrah kamraten añ ta rājya sous le règne de Jayavarman $\mathrm{V}$ (nous avons mentionné précédemment le cas de la cérémonie d'ouverture des yeux rapportée par l'inscription K. 1141, retrouvée près de Khorat).

Enfin et surtout, le récit de Sadāśiva n'est supporté à aucun moment par l'épigraphie antérieure au règne de Jayavarman IV pour faire remonter la naissance du culte du Devarāja à une date aussi haute que 802. Il n'y est tout simplement fait aucune allusion ${ }^{15}$. La stèle de Sdok Kak Thom, pas davantage qu'une autre inscription, n'est donc en mesure de remettre en cause le constat, formulé autrefois par G. Coedès, selon lequel la naissance du culte du dit Devarāja prend place à Koh Ker sous le règne de Jayavarman IV - étant entendu que cette fondation du Devarāja ne s'est pas faite ex nihilo et retravaille selon toute vraisemblance des éléments cultuels déjà existants.

On ne saurait donc considérer le contenu de la stèle de Sdok Kak Thom, sans qu'il soit soumis à un rigoureux examen critique (somme toute assez classique). On prêtera ainsi attention non seulement à la date de sa rédaction, assez tardive, mais aussi au contexte très spécifique dans lequel elle s'inscrit. Datée du mois de février 1053 de n. è. (974 śaka), l'inscription est là gravée au début du règne de Udayādityavarman II (r. 1050-1066). Son objet est de commémorer une double fondation, dans laquelle le souverain est partie prenante (offrant lui-même un linga), en même temps que de réaffirmer des droits sur des terres dont les fondations, précise le texte, ont été détruites lors des troubles ayant marqué le règne précédent de Sūryavarman $I^{\text {er }}$ (r. 1002-1050). Le long rappel des fondations religieuses entreprises par les ancêtres de Sadāśiva comporte une évidente dimension politique : le passé se trouve ici transformé, amputé, aménagé dans un contexte de compétition propre au $\mathrm{XI}^{\mathrm{e}}$ siècle angkorien, contexte opposant pour le statut social et le pouvoir les membres des grandes maisons aristocratiques de l'époque.

15. L'évocation de la royauté divine d'Indra (devaräjya) dans la stèle de Preah Kô (K. 713 st. VI, 880 A.D.) ne saurait constituer ici une exception (contra Codès et Dupont 1943-46, p. 64). 
Le passé y est aussi, et sans aucun doute, préservé. Il n'est pas question, après avoir trop ignoré Koh Ker, de trop ignorer la stèle de Sdok Kak Thom. Nous avons simplement là une histoire plus complexe à écrire, évidemment moins figée, moins linéaire que celle écrite dans le sillon étroitement tracé par Sadāśiva. Il ne fait aucun doute que le Prasat Thom d'un côté, la stèle de Sdok Kak Thom de l'autre, nous donnent à voir deux étapes d'une même histoire, celle d'un culte spécifique à Śiva, reconnu comme la divinité tutélaire de la royauté angkorienne : deux étapes qui sont non seulement séparées par plus d'un siècle, mais qui s'offrent surtout à l'analyse à travers des documents de nature radicalement différente.

Il est donc exclu de chercher à rabattre les enseignements de la stèle de Sdok Kak Thom sur l'analyse du Prasat Thom. Pour autant, il ne s'agit pas non plus de renoncer à intégrer l'une et l'autre dans une histoire commune du Devarāja. Sans avoir à confondre une telle histoire avec l'une des étapes de son évolution, il demeure ici légitime de s'interroger sur ce qui relie au fil de plus d'un siècle l'évocation, à Sdok Kak Thom, de pratiques centrées sur la figure de Tumburu et les spécificités, au Prasat Thom, du dispositif architectural et surtout iconographique mis en œuvre.

Certes, au préalable, il convient également de rappeler ces deux fortes mises en garde émises par les historiens du śivaïsme. (1) Les ouvrages tels que le Vịnāśikha ne fournissent qu'un cadre à la pratique concrète qui est, elle, reflétée dans les Paddhatis, les manuels d'application qui guidaient les procédures suivies par l'officiant : ces manuels visent à extraire "l'essence » de tel ou tel ouvrage mais sans s'interdire de s'ouvrir, si nécessaire, à d'autres sources (appartenant éventuellement à d'autres divisions du śivaïsme). En d'autres termes, la mention de tels ouvrages dans les inscriptions ne suffit pas à livrer la clé des rituels que nous étudions. (2) Les procédures décrites dans ces mêmes ouvrages (sélectionner un système de Mantra, la configuration d'une série de divinités, etc.) ne sont guère concernées par les aspects dits «publics» des pratiques religieuses (le Vịnăşikha ne comporte ainsi aucune procédure d'installation des images, pratișthā).

En conséquence, est-il suggéré, les pratiques rituelles que l'épigraphie invite à restituer et qui nous renvoient aux spécificités des différentes divisions de l'Atimārga ou du Mantramārga, peuvent n'avoir laissé aucune trace dans ce qui constitue la partie visible du temple - et qui retient en premier l'attention de l'archéologue -, à savoir son architecture et l'éventail plus ou moins vaste des images. 
La définition de ces programmes iconographiques relèverait davantage des laïcs que de la communauté des initiés (Sanderson 2003-04, p. $358,360,435-437,444)$.

On ne saurait ignorer ces vigoureuses mises en garde, mais les enseignements qui en sont tirés pour l'analyse iconographique prêtent peut-être là à discussion. On peut s'interroger en particulier sur la pertinence, en dehors de tout examen d'une communauté donnée, d'une distinction tranchée entre un monde privé des initiés et un monde public des laïcs. N'y a-t-il pas la place pour une voie moyenne, à bonne distance des deux approches qui nous sont proposées en guise d'alternative : l'une qui ne voit dans les images qu'un simple décalque des systèmes rituels, y compris les plus ésotériques, documentés par l'épigraphie ; l'autre qui n'envisage le temple et les images que comme un cadre neutre (et intangible) sans rapport avec la nature même du culte rendu dans le temple ${ }^{16}$ ? Ainsi que nous le proposions en introduction, une telle voie moyenne nous paraît, à tout le moins, susceptible d'apporter un éclairage renouvelé sur l'histoire du Devarāja et le programme iconographique du Prasat Thom de Koh Ker.

\section{Le programme iconographique}

L'une des grandes originalités de l'art de Koh Ker est de représenter en ronde-bosse ce que les artistes khmers réaliseront ailleurs en bas-reliefs. Le Prasat Thom, auquel on peut ajouter le Prasat Chen (son pendant viṣnuïte à Koh Ker) ${ }^{17}$, a assurément abrité quelques-uns des groupes sculptés les plus exceptionnels de la statuaire khmère.

Selon le principe, évoqué plus haut, d'une manifestation graduée du divin vers la périphérie, cette iconographie en ronde-bosse se déploie dans les pavillons d'entrée des enceintes extérieures du sanctuaire. Une série d'identifications inédites a permis d'en renouveler entièrement la compréhension même si, comme nous allons le voir, subsistent de nombreuses interrogations. Nous allons nous intéresser en particulier à trois de ces pavillons, ainsi qu'à l'une des deux tours annexes érigées à l'extérieur de la troisième enceinte. L'ensemble de ces édifices abritait une vingtaine d'images.

16. Voir, à ce sujet, Filliozat 2007, p. 99

17. Bourdonneau 2011, p. 128. 


\section{LE JUGEMENT DES MORTS}

La meilleure introduction à ce riche programme iconographique nous est fournie par deux stèles anépigraphes, provenant l'une du site d'Angkor Thom (fig. 4-5), l'autre du temple de Vat Phu (fig. 6-7), le grand lieu saint du Cambodge ancien, aujourd'hui dans le Sud du Laos. Stylistiquement, les deux stèles peuvent être datées du $\mathrm{X}^{\mathrm{e}}$ siècle. Telles que nous les interprétons, toutes deux livrent la reproduction en miniature du programme réalisé à une échelle monumentale au Prasat Thom. Plus exactement, les deux faces de chacune de ces stèles reproduisent le dispositif iconographique des deux gopura (est et ouest) de la seconde enceinte du Prasat Thom.

Nous commencerons par la description du groupe sculpté du gopura II Est et ce que nous désignerons comme la face A de chacune des deux stèles. Sur cette face, nous reconnaissons tout d'abord deux divinités aisément identifiables : au centre ou à droite, Indra sur l'éléphant Airāvata et, à gauche, Yama, roi des enfers ainsi que dieu de la mort et des morts, assis sur son buffle et tenant la massue (le daṇda).

Sur la stèle de la région de Vat Phu, dont le décor est le plus soigné et le plus riche, apparaissent quatre autres personnages accroupis. Ceux placés aux extrémités offrent les mêmes traits terribles qui les désignent volontiers comme deux assesseurs de Yama : coiffure dénouée à grosses mèches, sourcils froncés, yeux ronds exorbités, crocs dépassant de la commissure des lèvres. La dernière arcature, à la droite d'Indra, abrite encore deux autres personnages : le plus grand des deux se présente comme un personnage coiffé d'un diadème et présentant devant lui un objet de forme allongée et strié horizontalement, que l'on peut identifier comme un manuscrit. Ce personnage, nous le retrouvons sur la stèle d'Angkor Thom, au pied de Yama, tenant également un objet dans sa main droite, difficilement lisible ici. Sur la stèle de Vat Phu, figure à ses côtés un dernier personnage, représenté à une échelle nettement plus réduite. Tête nue, ses bras sont rejetés vers l'arrière.

C'est bien sûr l'examen de chacune de ces positions et de chacun de ces gestes qui livre les premières clés de compréhension de cette image. Elle peut être définie tout d'abord comme une scène de jugement des morts présidée par Yama. La comparaison avec la stèle d'Angkor Thom établit clairement que le personnage représenté à la droite d'Indra à Vat Phu n'est pas tourné vers celui-ci mais vers 


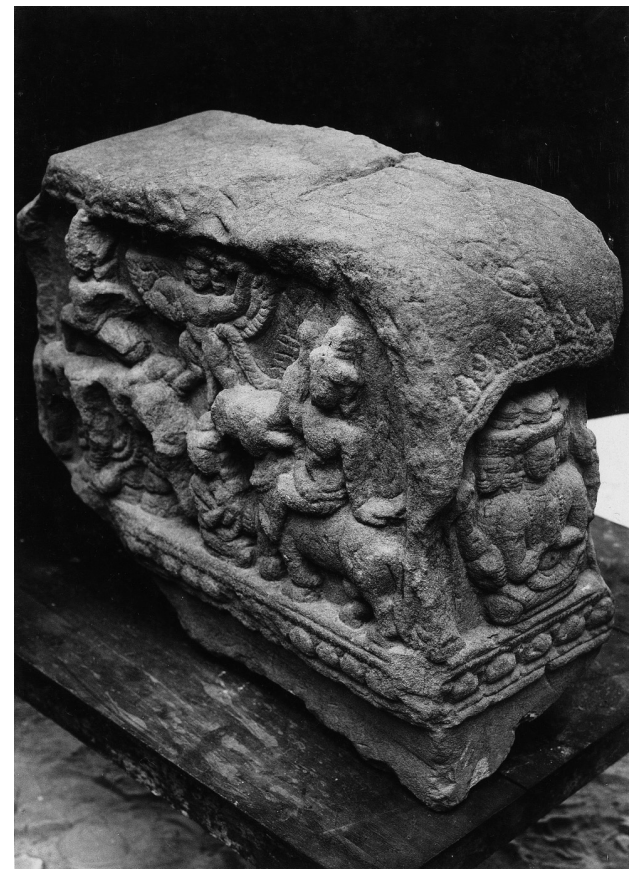

FIG. 4. - Stèle anépigraphe d'Angkor Thom, face A (Archives photographiques de l'EFEO [02288]).

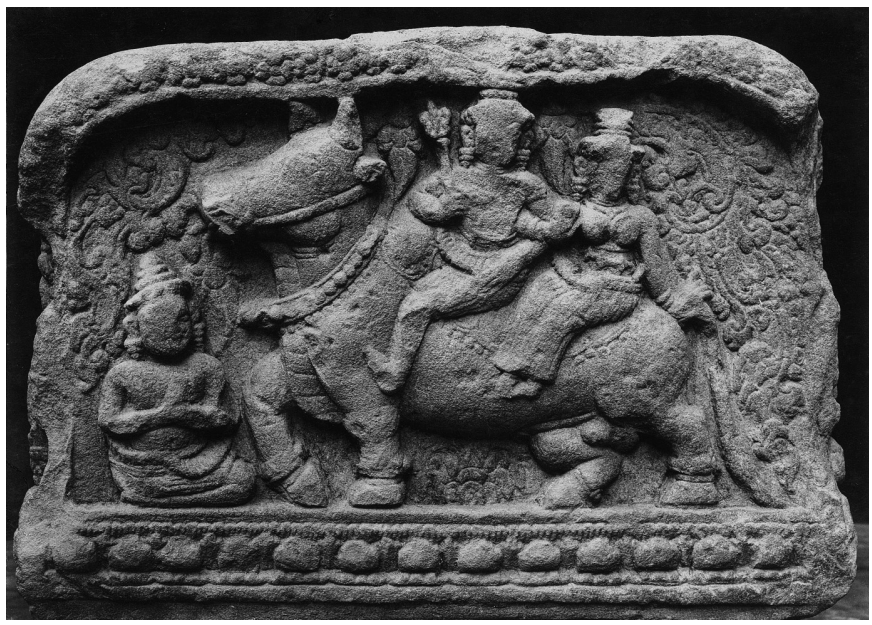

FIG. 5. - Stèle anépigraphe d'Angkor Thom, face B (Archives photographiques de l'EFEO [02290]). 


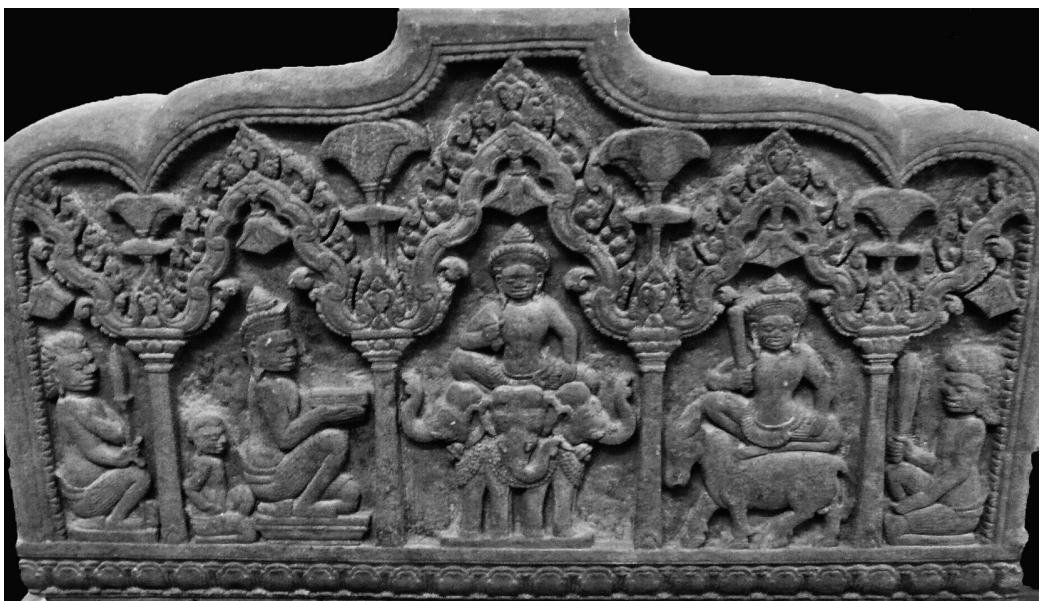

FIG. 6. - Stèle anépigraphe du temple de Vat Phu, face A (ph. Ch. Hawixbrock).

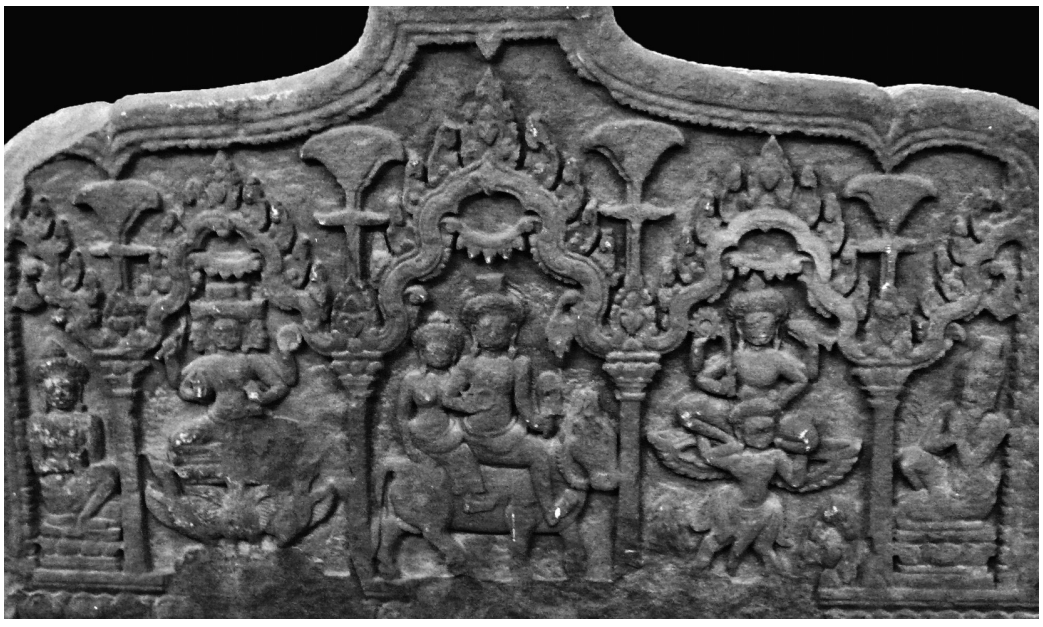

FIG. 7. - Stèle anépigraphe du temple de Vat Phu, face B (ph. Ch. Hawixbrock).

Yama. La présence des assesseurs du dieu de la mort aux extrémités suggère également que l'architecture représentée est bien celle du palais $(s a b h \bar{a})$ du roi des enfers. Indra n'est pas tant représenté au centre que devant ou au-dessus. 
Si l'interprétation de la figure tournée vers Yama ne va certainement pas de soi, elle se ramène, à nos yeux, à l'alternative suivante.

Une première hypothèse est qu'il s'agit de Citragupta, le scribe et l'archiviste au service de Yama, « qui garde la diversité (des actions bonnes et mauvaises des hommes) ${ }^{18}$. Le manuscrit présenté au dieu des morts serait donc le registre consignant les faits accomplis par le défunt que l'on devrait, dès lors, identifier avec le personnage représenté au pied de Citragupta. La position des bras, rejetés en arrière, de ce personnage se comprend aisément comme celle de bras liés dans le dos. Nous la retrouvons à l'identique dans la célèbre galerie dite des enfers à Angkor Vat dans une autre scène de jugement des morts, beaucoup plus célèbre celle-ci, lointaine héritière de ce que nous découvrons ici. Encadré par les assesseurs menaçants de Yama, les membres entravés, il nous faudrait alors comprendre que le défunt ainsi représenté figure parmi des damnés destinés aux supplices des enfers.

Sans doute est-ce là l'hypothèse qui paraît s'imposer le plus spontanément, car elle repose en apparence sur une association bien documentée dans les sources textuelles, celle de Citragupta et de son registre. Elle ne pose pas moins de sérieuses difficultés. Le geste même esquissé par ce supposé Citragupta, n'est pas la moindre d'entre elles. Car on ne voit nulle part ailleurs au Cambodge, et sans doute plus largement dans le monde indianisé, celui-ci remettre ainsi à Yama le registre enregistrant les actes du défunt. Tout au contraire, pourrait-on dire, si Citragupta assiste Yama, c'est précisément que lui revient en propre la charge d'énoncer le récit des actions humaines. Entre autres objections, l'on pourrait également s'étonner que Citragupta apparaisse ici sans la figure farouche de Dharma qui l'accompagne traditionnellement (et qui ne saurait être confondu ici avec l'un ou l'autre des gardiens représentés sous les arcatures latérales).

Si l'hypothèse de Citragupta doit être écartée et si, partant, on voit difficilement quel autre personnage de la suite de Yama pourrait jouer un tel rôle, il nous faut envisager une toute autre hypothèse, sans doute inattendue mais plus satisfaisante : en lieu et place de Citragupta, ce serait le défunt lui-même qui se présenterait à Yama et qui soumettrait au jugement du dieu le récit de ses actes. Dès lors, ce personnage qui, à Vat Phu, porte un diadème et

18. Cf. Malamoud 1997, p. 97 (se référant au Śabdakalpadruma). 
un couvre-chignon, a toutes les chances d'être le roi lui-même, de sorte que les trois arcatures centrales de la stèle représenteraient les souverains des trois mondes - une triple souveraineté ellemême englobée par la souveraineté suprême de Śiva "Seigneur des trois mondes » (représenté ici au revers de chacune des stèles, cf. infra).

Quel peut être ce récit sinon celui des actions dharmiques du souverain, telles que celles-ci sont célébrées dans les panégyriques des inscriptions mais aussi illustrées dans le décor même de la stèle ? Car on devine que le personnage au pied de ce que nous interprétons comme le souverain doit être ici compris comme la figure de l'ennemi du dharma (et de l' " ordre » que dénote une telle notion) soumis par le roi. Sa mise à mort semble même déjà se préparer : ainsi, le personnage farouche à la gauche de Yama a certainement pour vocation de réaliser cette mise à mort. Tandis que son acolyte tient une lance et pose sa main droite sur l'épaule gauche dans une attitude de fermeture du corps, celui-ci tient une épée à large lame et pose sa main gauche sur sa cuisse dans une posture plus ouverte à l'action.

Ce faisant, le souverain se présente à Yama, juge des morts mais aussi dharma-rāja, comme étant lui-même, à l'instar du dieu, un autre roi du dharma, ayant pour fonction de veiller à ce que l' " ordre (socio-cosmique) » soit préservé en châtiant quiconque le négligerait (Malamoud 2002, p. 25).

Examinons à présent le groupe sculpté du gopura II est du Prasat Thom (fig. 8). Il réunit huit images. Ces statues ont malheureusement beaucoup souffert du pillage et leurs fragments ont été déplacés à de nombreuses reprises depuis la première description du site par Louis Delaporte à la fin du XIX ${ }^{\mathrm{e}}$ siècle. L'inventaire des fragments subsistant in situ, le relevé précis du dallage et des marques au sol signalant l'emplacement originel des statues, ainsi que la lecture de la planche publiée par Louis Delaporte ${ }^{19}$, ont permis de restituer l'organisation de ce groupe sculpté (fig. 9).

Dans le bras sud du gopura trônait Yama sur son buffle. À sa droite, prenaient place Sūrya et Candra, le Soleil et la Lune, dont la présence ici évoque irrésistiblement les stances comminatoires des inscriptions : ceux qui violent les fondations religieuses iront aux

19. Cf. Delaporte 1880, p. 96. 


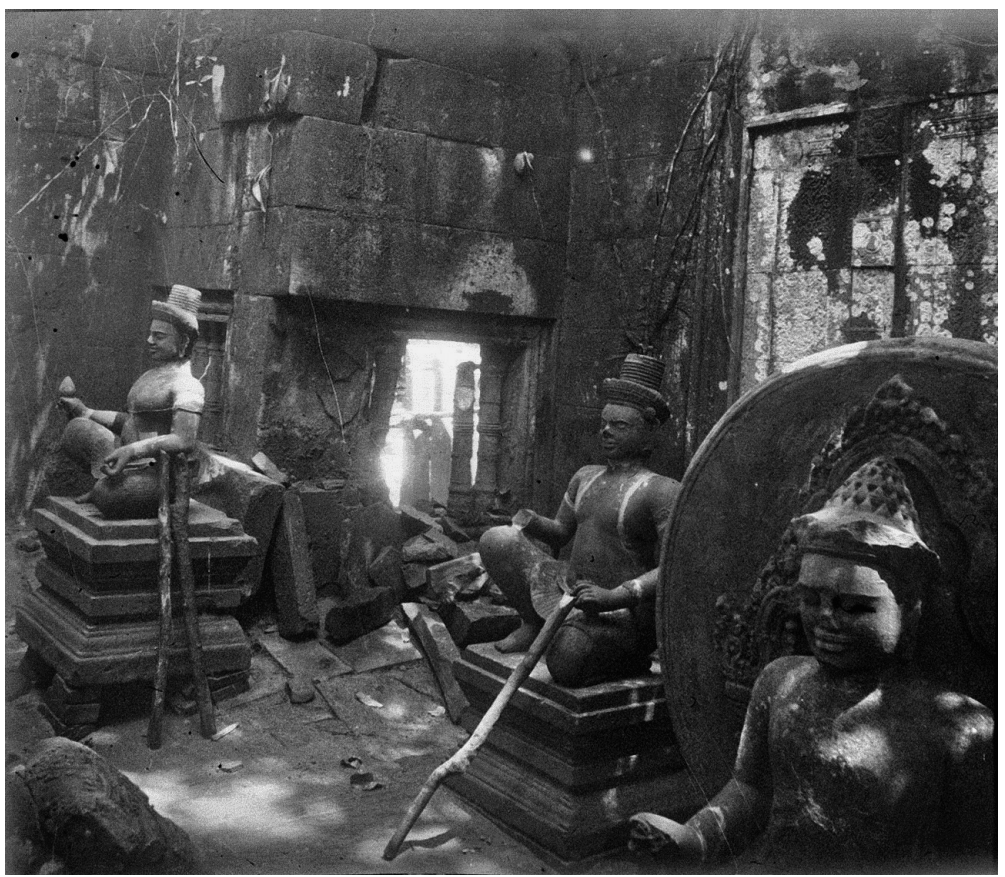

FIG. 8. - Gopura II est du Prasat Thom

(Archives photographiques de l'EFEO, Fonds Parmentier [PAR02776]).

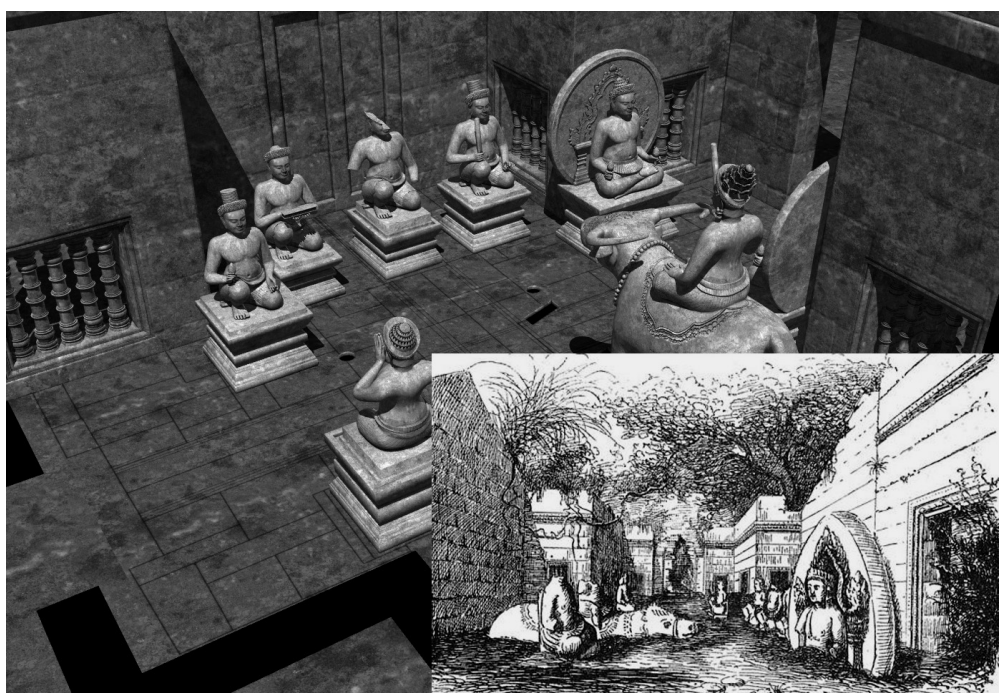

FIG. 9. - Gopura II est du Prasat Thom, proposition de restitution du groupe sculpté (réalisation : Grez productions) et planche de Louis Delaporte. 
enfers « aussi longtemps que la lune et le soleil dureront $»^{20}$. En face de Yama, et encadrant deux statues sur lesquelles nous reviendrons ensuite, nous reconnaissons les deux assistants bien connus de Yama, le scribe Citragupta et Dharma, ce dernier aux traits ici légèrement plus farouches.

Parmi les trois statues restantes, un intéressant personnage de bossu était placé à la gauche du roi des morts. Ce bossu se retrouve dans différents grands sanctuaires des $\mathrm{XI}^{\mathrm{e}}$ et $\mathrm{XII}^{\mathrm{e}}$ siècles. Il est ainsi attesté au Phnom Chisor, à Vat Phu ou encore à Preah Vihear, soit, là encore, dans les grands sanctuaires de lieux saints qui nous paraissent, à bien des égards, les héritiers du Prasat Thom de Koh $\mathrm{Ker}^{21}$. Nous retrouvons également des personnages de bossu (ainsi que Yama) dans les grands monuments du style du Bayon. Dans ces exemples plus tardifs, le bossu est représenté tenant un trébuchet. Dès lors que l'association avec Yama est établie, il est difficile de ne pas songer que la petite balance en question était un instrument du jugement des morts. Cependant, au Prasat Thom, le bossu a les mains jointes et ne tient aucune balance. Une hypothèse envisageable, que nous ne proposons que sous toutes réserves, est que la balance était, à l'époque de Jayavarman IV, l'attribut d'un personnage distinct qui pourrait être le personnage se tenant face à Yama (et dont l'image offre ce point commun avec celle du bossu de comporter une cuve à ablution tandis que les autres statues en sont dépourvues et sont monolithes avec leur piédestal).

Reste une huitième statue : la trace d'arrachement laissée par le bras sur la cuisse droite, l'orientation de ce bras, l'absence d'une trace semblable sur la cuisse gauche (plus basse) suggèrent que le personnage en question tendait les mains sans les avoir aussi hautes que le bossu (qui les a jointes). Sa coiffure singulière - un chignon aplati qui paraît le résultat d'une volonté de traitement plus réaliste - pointe dans la même direction : selon toute vraisemblance, il s'agit de l'équivalent en ronde-bosse du personnage royal se présentant devant Yama sur la stèle de Vat Phu, auquel cas il ne pourrait ici s'agir que du souverain Jayavarman IV en personne (fig. 10).

Le dieu Indra ne figure pas dans ce groupe sculpté. Cependant, nous le retrouvons dans la tour sud à l'extérieur de la troisième

20. Nous retrouvons le Soleil et la Lune aux côtés de Yama (flanqué de Dharma et Citragupta) sur le décor sculpté de la stèle de Stung Chrap (K. 693, Musée national du Cambodge : inv. Ka 185).

21. Plusieurs bossus sont également attestés sur les sites rupestres des Monts Kulen qui offrent aussi de nombreuses affinités avec ces sites de lieux saints. 


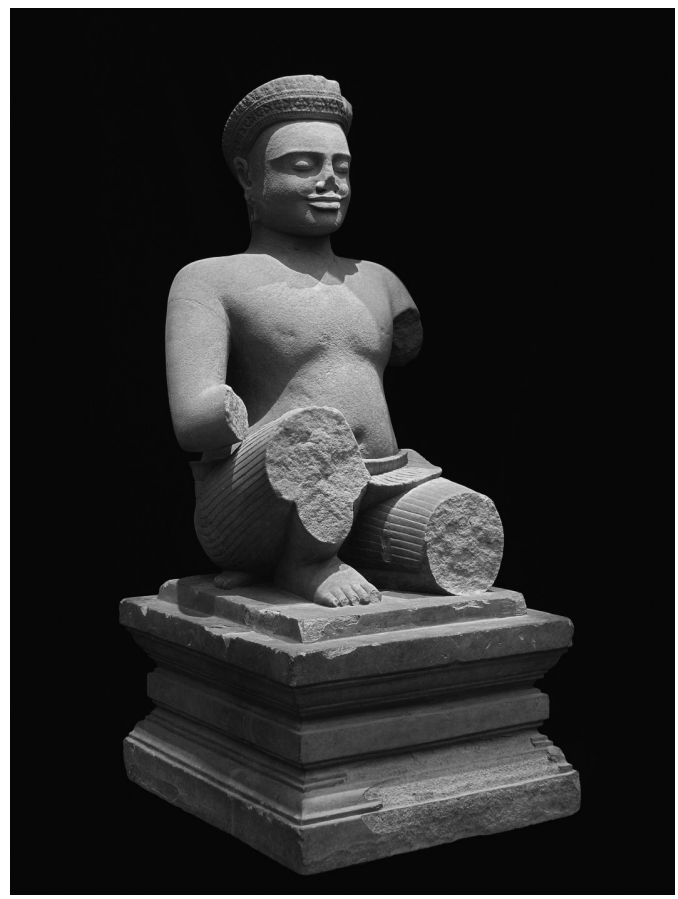

FIG. 10. - Statue supposée de Jayavarman IV, gopura II est du Prasat Thom (ph. P. Baptiste - musée Guimet).

enceinte. En accord avec la stèle, il précède bien l'assemblée de Yama. Quant au Garuda de la stèle d'Angkor Thom, il se retrouve bien aussi à Koh Ker, de part et d'autre de la chaussée d'accès devant la seconde enceinte, dans des proportions monumentales.

Nous évoquerons plus brièvement les quatre images du gopura II ouest et l'iconographie correspondante sur les faces B des stèles de Vat Phu et d'Angkor. Elles se rapportent à différents moments du Kirātārjunīya. Il s'agit là d'un épisode fameux du Mahābhārata. Lors de l'exil des frères Pāṇạa dans « la forêt », Arjuna, figure royale par excellence de l'épopée, se rend au ciel d'Indra mais il doit, pour y arriver, pratiquer de dures austérités et se battre à mains nues avec Śiva déguisé en «montagnard». Nous reconnaissons cette lutte de Śiva-kirāta et d'Arjuna parmi le groupe sculpté du gopura II ouest (fig. 11), mais elle n'apparaît pas sur les stèles. Śiva prend naturellement le dessus et transforme Arjuna, 


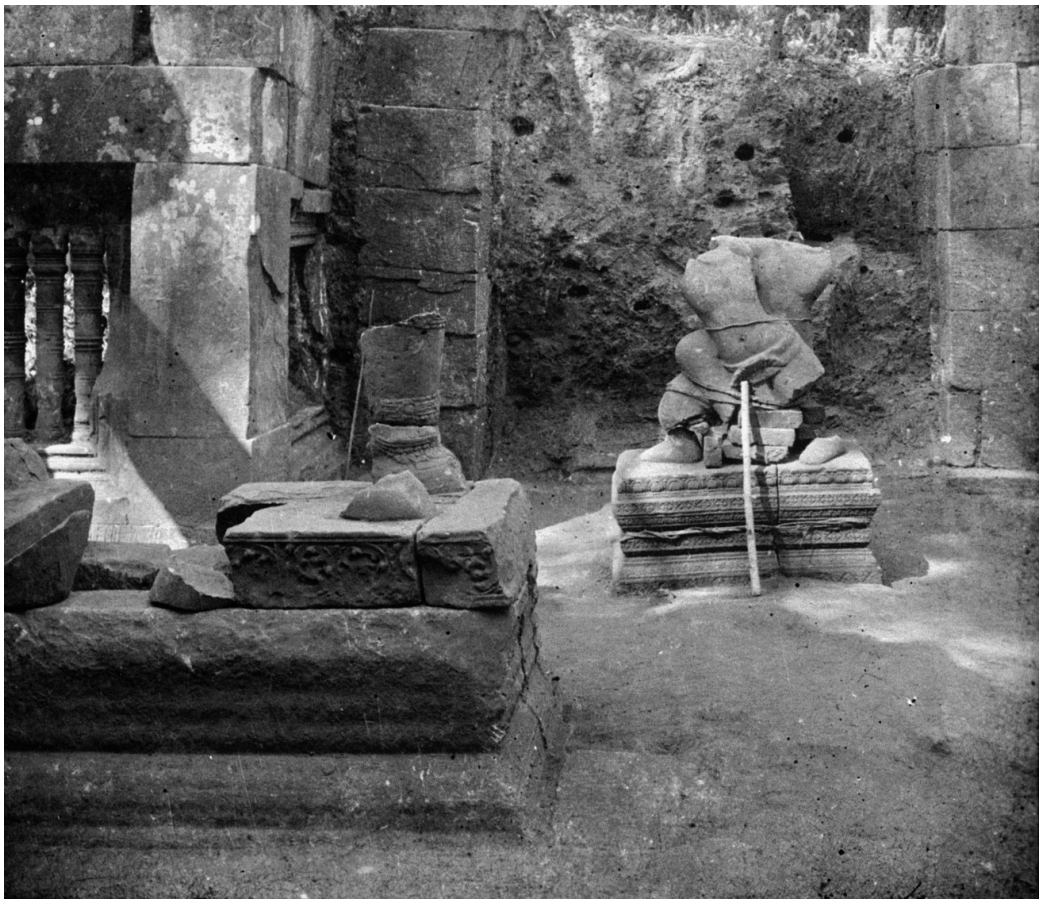

FIG. 11. - Kirātārjunīya, gopura II ouest du Prasat Thom (Archives photographiques de l'EFEO, Fonds Parmentier [PAR02681]).

précise le Mahäbhärata, en " boulette » de nourriture, semblable aux boulettes funéraires (Biardeau 2002, p. 462). Ramené à la vie, Arjuna reconnaît le dieu et lui rend un culte. Siva lui donne alors l'arme brahmaśiras et les dieux des points cardinaux font de même, en donnant à Arjuna leurs armes respectives. Au Prasat Thom comme sur la stèle de Vat Phu, c'est probablement Arjuna qu'il faut également reconnaître sous les traits d'un ascète barbu tourné, les mains jointes, vers Umāmaheśvara sur le taureau (tandis que, dans chacune de nos représentations, Śiva est lui-même flanqué par Viṣnu et Brahmāâ) ${ }^{22}$. Enfin, le personnage portant un diadème et un couvre-chignon qui adopte la même attitude à l'autre extrémité de la stèle de Vat Phu pourrait être ici une seconde 
représentation du roi qui, par le jeu de la symétrie, serait pleinement assimilé à son modèle épique, Arjuna.

Sur chacune des faces, comme dans chacun des gopura, les images se répondent ainsi les unes aux autres. Cet écheveau de relations entre les images, relations d'homologie, de dédoublements ou de redoublements, s'étend d'une face ou d'un gopura à l'autre. Sur la stèle de Vat Phu, la représentation des souverains de chacun des mondes s'adosse, avons-nous vu, à celle de Śiva luimême «Seigneur des trois mondes ». Au Prasat Thom, la représentation du Kirātārjunīya nous semble mettre l'accent sur une autre dimension de la juxtaposition des images. Entre Arjuna réduit à l'état de « boulettes funéraires » et le souverain se présentant devant Yama, ne faut-il pas effet reconnaître le développement iconographique d'un même thème mêlant sacrifice et mort-renaissance ?

Revenons à l'examen du gopura II est et de la face A de la stèle de Vat Phu. Si le souverain se présente devant l'assemblée de Yama, c'est bien qu'il y apparaît comme un trépassé. Mais peut-il être mort et représenté tout à la fois, ainsi que nous l'avons suggéré, agissant ici-bas comme un autre Yama, avec toute la sévérité qu'exige la préservation du dharma parmi les hommes ? La contradiction se résout d'elle-même si le souverain n'est mort que rituellement - la scène se déroulant entre deux mondes distincts mais liés par le rituel. N'est-ce pas précisément en ce sens qu'il faut également interpréter l'évocation de la mise à mort (effective celleci) de la figure de l'ennemi du dharma ? L'analyse que nous en avons proposée plus haut est évidemment incomplète. Si elle est l'illustration de la fonction répressive de la royauté, son sens n'estil pas aussi celui d'un sacrifice (en ne perdant pas de vue le contexte ici śivaïte dans lequel s'inscrit notre dispositif) ? Plus exactement, l'un et l'autre aspect ne se ramènent-ils pas à la même dimension de l'action dharmique du souverain ? Le sacrifice (yajña) figure au premier rang des rites par lesquels s'effectue le dharma; or, ce que le sacrifiant donne dans le sacrifice, c'est lui-même (Malamoud 2002, p. 30-31). Le sacrifié, la matière du sacrifice, n'en est que le substitut. En livrant à la mort les êtres vils de la « surface d'enbas » dont il est le souverain, celui-ci met ainsi doublement en œuvre le dharma. Les vies des ennemis vaincus fournissent le substitut de sa propre personne se sacrifiant. Sans doute est-ce là l'occasion de rappeler ici que la pratique du sacrifice humain 
(offrant toujours une dimension royale) est clairement attestée dans l'histoire khmère, à la fois en amont et en aval de la période angkorienne ${ }^{23}$.

Le Cambodge plus récent, mais aussi l'Inde, fournissent également de précieux éléments de comparaison pour mieux comprendre cette mort-renaissance rituelle que nous venons d'évoquer. Les indianistes songeront peut-être ici à la relation établie entre Yama et Naciketas dans le récit de la Kaṭhopanișad: Naciketas subit une mort rituelle et reste dans la «maison de la Mort » durant « trois nuits » qui pourraient ici avoir valeur de régression temporaire au chaos, "afin d'être transformé et de connaître une nouvelle naissance d'ordre mystique » (Chenet 2004, p. 584). La relation à Yama est de nature initiatique ; ce n'est qu'après cette épreuve subie avec succès que Yama dispense à l'initié un enseignement plus ésotérique. Le récit permet d'entrevoir comment, dans nos sources, le jugement des morts est peut-être à comprendre comme un concept liminal, symbolisant le franchissement d'un seuil entre deux mondes ou deux états ${ }^{24}$. Certes, la référence au Kaṭhopanișad peut paraître bien éloignée du Cambodge angkorien ; toutefois, elle l'est déjà un peu moins si l'on songe qu'un récit assez semblable, où Yama apparaît comme le dépositaire du salut, se retrouve au Cambodge dans le corpus des textes qui définissent les spécificités d'un bouddhisme khmer très différent de l'orthodoxie cinghalaise (Bizot 1989, p. 21). Enfin, dans le sens de cette interprétation d'une mort rituelle du roi suivant le schéma d'une initiation, les anciennes cérémonies dites du «troisième mois », auxquelles nous avons déjà fait allusion, méritent peut-être d'être à nouveau évoquées comme un lointain écho à ce que nous voyons à Koh Ker : le souverain se retirait pendant trois jours dans son palais ; un roi temporaire était nommé et prenait sur lui le malheur. Dans son cortège, peut-on relever incidemment, figuraient en particulier deux bossus (Leclère 1917, p. 292).

\section{LA DANSE TERRIBLE DE ŚIVA}

Le gopura III est, édifice aux dimensions colossales désigné aujourd'hui comme le Prasat Kraham, abritait l'ensemble de rondesbosses le plus imposant du sanctuaire. Ce groupe sculpté était aussi

23. Pour un premier aperçu des données disponibles, cf. Chandler 1996, p. 119-135 et PoréeMaspéro 1962, p. 246-248 et p. 275-282.

24. Sur les phénomènes de liminalité, voir les travaux de Victor Turner cités dans Assmann 2010, p. 71 
le premier que rencontrait le dévot progressant vers l'intérieur du monument. Il mériterait à lui seul d'y consacrer de longs développements. Et, là encore, définir la part de ce qui est établi, de ce qui peut être raisonnablement supposé, ou de ce qui relève davantage de la spéculation, sera la matière d'un équilibre délicat à trouver. Nous nous contenterons d'évoquer, en simplifiant beaucoup, ce qui permettra de proposer une première conclusion au survol que nous avons souhaité esquisser ici du Prasat Thom.

Cinq images, disposées en quinconce, prenaient place dans ce gopura. Au centre se dressait la statue monumentale de Śiva-Rudra, à cinq têtes et dix bras, dansant sur un serpent dont le corps s'enroulait autour du piédestal (fig. 12). Ses proportions dépassaient le double de la taille humaine. Six de ses mains ont été retrouvées. Parmi les attributs du dieu, nous reconnaissons le lasso, le bouclier, la massue et la calotte crânienne. Les quatre sculptures qui entouraient la statue de Śiva, étaient implantées selon deux principes combinés : les formes féminines en avant des divinités gardiennes masculines ; les formes paisibles à la droite du dieu, les formes terribles à sa gauche. Sont ainsi représentés deux aspects complémentaires de la déesse, reflet de l'ambivalence de la danse de Śiva, comme nous allons y revenir. Umā, à la droite du dieu, est représentée elle-même dansante. Cāmuṇạa, la forme terrible et sanguinaire de la déesse, identifiable à la chouette sculptée sur chacune des faces de son piédestal, est représentée assise et tenait dans sa main une tête décapitée (fig. 13) ${ }^{25}$. Dans la partie ouest de l'édifice, nous reconnaissons les deux « chefs de gaṇa » (ganeśvara), Nandikeśvara et Mahākāla (les troupes de Śiva, gaṇa, étant elles-mêmes représentées sur les échiffres des différents gopura). Le premier présente un troisième œil à l'instar de Śiva et jouait probablement du tambour rythmant ainsi la danse du dieu; le second reproduit les mêmes traits farouches que Cāmuṇụā.

Envisageons à présent, mais cette fois-ci de façon beaucoup plus précise, l'hypothèse qui fut notre point de départ : le groupe sculpté du Prasat Kraham peut-il être mis en relation, d'une quelconque façon, avec le culte du Devarāja ? Dès lors que l'on a renoncé à la thèse des « deux niveaux de réalité », la réponse va, pour ainsi dire,

25. Nous retrouvons Cāmuṇ̣̂ā avec la chouette comme monture sur les frises dites des «sept Mères » (sapta-mātrkā $)$. 


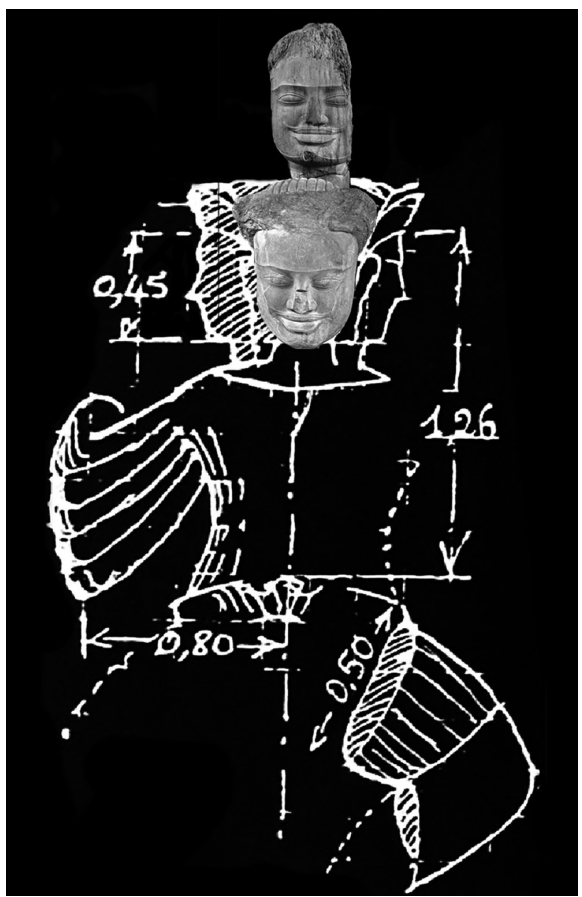

FIG. 12. - Śiva dansant, gopura III est du Prasat Thom (Croquis G. Groslier et ph. Atelier de restauration du Musée national du Cambodge).

de soi. L'image de Śiva dansant, érigée à cet emplacement du sanctuaire, s'intègre pleinement dans le mouvement d'expansion cosmique du temple évoqué plus haut. Le jeu sur les proportions des édifices s'applique également aux images que ces derniers abritent. La danse colossale de Śiva n'est autre que la manifestation visible, agissante au sein des mondes, du linga de Śiva Tribhuvaneśvara installé dans la petite tour centrale - ce même Śiva Tribhuvaneśvara identifié au vrah kamraten añ jagat ta rājya des inscriptions khmères et, partant (certes de façon plus indirecte), au Devarājalkamratén jagat ta rāja célébré par Sadāśiva.

Il y a là un intéressant parallèle à établir avec l'évolution du motif de la danse de Śiva en Inde même et dans le monde tamoul en particulier (déjà évoqué à propos de l'architecture). Sensiblement à la même période que le règne de Jayavarman IV, la représentation de la danse de Śiva s'impose progressivement dans les temples cōla comme l'icône royale par excellence de la dynastie régnante (Kaimal 


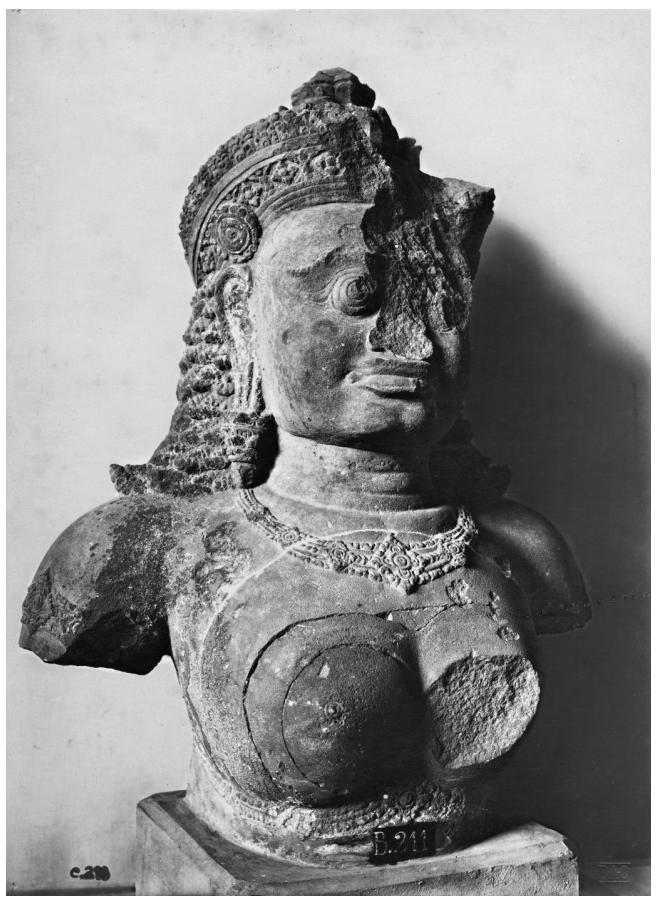

FIG. 13. - Buste de Cāmundā provenant du gopura III est du Prasat Thom (Archives photographiques de l'EFEO [15965]).

1999). Nous retiendrons deux aspects de la construction iconographique de cet emblème dynastique. Le premier est l'existence de " marqueurs iconiques », attributs ou figures auxiliaires, qui associent la danse de Śiva étroitement aux thèmes de la mort, de la crémation et de la destruction (destruction du cycle des existences comme du cycle cosmique). De façon plus précise, et surtout plus spécifique, les temples cōla du $\mathrm{X}^{\mathrm{e}}$ siècle nous donnent à voir ce moment précis où d'une danse sur la mort, la manifestation de Śiva évolue en une danse de la victoire. Elle n'en demeure pas moins une danse terrible, qui a toujours partie liée avec la mort, mais où les emblèmes de la destruction sont ici brandis comme autant de trophées d'un dieu conquérant et triomphant. Un tel motif est d'autant plus propice à l'exaltation de la royauté que ses propriétés formelles, et c'est là le second aspect qui nous intéresse, se prêtaient de façon remarquable à l'expression d'un pouvoir en expansion. L'explosion d'énergie rendue par la multiplication des bras et leur 
distribution circulaire, s'interprète aisément comme un mouvement rayonnant dans toutes les directions.

Si les divergences sont assez nombreuses avec ce que nous voyons au Prasat Thom, il demeure que les bâtisseurs de Koh Ker, et ceux qui s'en inspireront, nous semblent proposer une lecture assez semblable de la danse de Śiva. À bien des égards, une telle lecture y apparaît même plus explicite (ce qui, paradoxalement, est à interpréter comme le signe d'une réelle singularité vis-à-vis des réalisations indiennes). La danse du dieu est tournée ici vers l'Est et domine de façon majestueuse l'axe majeur du temple, là où elle fait généralement face au Sud dans le monde cōla. Ce faisant, comme nous l'avons vu, l'image khmère de Śiva dansant s'intègre parfaitement dans la structure concentrique et axée du temple : le mouvement d'expansion du divin se trouve ainsi suggéré et par l'architecture et par la danse du dieu. Que cette danse victorieuse du « Seigneur des mondes » demeure associée au thème de la mort et de la destruction, il suffit pour s'en convaincre de considérer la présence inquiétante de Mahākāla et surtout de Cāmuṇḍā au pied de Śiva ${ }^{26}$. Si les deux figures paraissent, au premier abord, rivaliser avec le voisinage de Nandikeśvara et d'Umā, la symétrie entre la droite et la gauche du dieu n'est toutefois pas parfaite. Cāmuṇạā assiste à la danse de Śiva en spectatrice, pourrait-on en dire, tandis qu'Umā l'accompagne et reproduit les mêmes pas que son époux, telle un autre «Seigneur de la danse »(nātyeśvara ${ }^{27}$. La complexe relation hiérarchique entre les deux dimensions de la danse observée dans le monde cōla se trouve respectée.

Il existe ainsi une remarquable convergence entre le dossier épigraphique et le dossier iconographique du Prasat Thom. Nous pouvons conclure non seulement que la danse de Śiva est la manifestation visible du vrah kamraten añ jagat ta rājya, soit Śiva en tant que «Seigneur des mondes» de ce royaume-ci (le royaume de Jayavarman IV), mais qu'elle propose de cette tutelle divine une image concrète et particulièrement expressive.

Un pas supplémentaire peut-il être franchi en identifiant le Śiva dansant du Prasat Thom avec le Śiva Tumburu auquel nous renvoie

26. Il faudrait là tout autant évoquer la juxtaposition, au sein du sanctuaire, de la danse de Śiva et du jugement des morts.

27. Sur la désignation de Śiva et de la déesse comme deux nātyeśvara (ainsi que sur la danse de Śiva dite « terreur des serpents ») voir Filliozat 1981, p. 72. 
la stèle de Sdok Kak Thom ? Nous pouvons d'emblée relever que les textes scripturaires connus ne semblent faire aucune allusion à une danse de Tumburu et l'entourage de celui-ci n'est évoqué que de façon assez lointaine par les images qui accompagnent celle de Śiva au Prasat Kraham. La réponse ne peut donc a priori être que négative, mais nous avons vu que l'enjeu véritable est ailleurs : non dans une stricte identification mais dans le relevé des éléments d'une histoire commune. Dans cette perspective, le travail de comparaison a davantage à offrir qu'une réponse aussi tranchée. Sans reprendre terme à terme les descriptions de l'une et de l'autre divinité, convenons de ce constat prudent : entre le Śiva Tumburu du Vīnāśikha, dieu guérisseur et protecteur, source de pouvoirs et de vie nouvelle, et le Śiva «Seigneur des mondes » de Koh Ker, dieu tutélaire tel que nous le fait connaître le dispositif du Prasat Thom - dispositif qui intègre dans le sillage de la manifestation dansante et victorieuse du dieu, une iconographie originale dans laquelle nous voyons le roi, ou ses substituts, sacrifier et se sacrifier pour mieux renaître et obtenir de Śiva des armes aux pouvoirs miraculeux -, la distance à parcourir n'est pas si grande. Elle ne saurait difficilement l'être moins. Car ce sont précisément, entre le Prasat Thom et le récit de la stèle de Sdok Kak Thom, deux époques, deux « usages », deux regards différents à travers lesquels nous percevons le culte du Devarāja.

L'objet des travaux à venir sur le Devarāja ne sera donc pas, à notre sens, de chercher à réduire davantage cette distance mais, d'une certaine façon, de mieux la documenter. Il s'agira de s'interroger sur les étapes intermédiaires de l'histoire de ce culte, entre le moment fondateur du règne de Jayavarman IV et celui de sa mise en récit dans la stèle de Sdok Kak Thom. En substituant le roi au royaume, la transformation, au début du $\mathrm{XI}^{\mathrm{e}}$ siècle, de l'expression vrah kamraten añ ta rājya en vrah kamratén añ ta rāja constitue assurément une étape importante. Il serait précieux de pouvoir déterminer quelles furent les formes architecturales et iconographiques prises par le culte du Devarāja à l'époque de cette transformation. Là encore, on évitera de conclure de façon trop hâtive que les sources font complètement défaut. La postérité, au-delà du règne de Jayavarman IV, du motif de la danse de Śiva est à reconsidérer à la lueur de son association au Prasat Thom avec le culte du vrah kamraten añ ta rājya. Sous le règne de Sūryavarman $\mathrm{I}^{\mathrm{er}}$, à cette même époque où, selon toute vraisemblance, le culte du Devarāja se fixe selon la forme documentée par la stèle de Sdok 


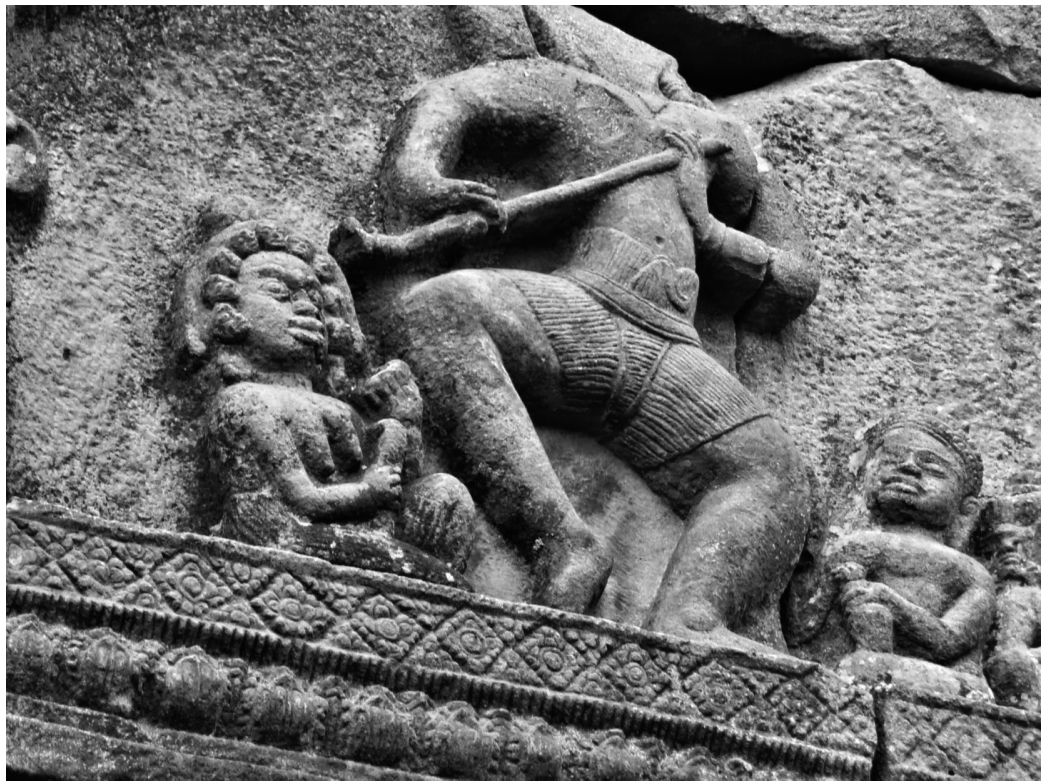

FIG. 14. - Śiva viñādhara et déesse coiffée de têtes décapitées, fronton est du gopura I est du temple du Phnom Chisor (ph. auteur).

Kak Thom, nous retrouvons la danse de Śiva aux places d'honneur des sanctuaires de Preah Vihear, de Vat Ek, de Vat Baset et du Phnom Chisor (fig. 14) : tous consacrés à Śiva désigné comme kamraten jagat, "Seigneur des mondes », et comme « Seigneur » du roi, Süryavarmeśvara (Seigneur du roi et non du royaume, mais démultiplié et érigé dans les différentes provinces de ce même royaume). Simple coïncidence ou non, le dieu dansant arbore à présent cette même vīnā à laquelle, quelques années plus tard, renvoie la mention du Viñăśikha dans la stèle de Sdok Kak Thom.

Quoi qu'il en soit de ce rapprochement, que l'on pourra juger bien fragile, subsiste le constat selon lequel le groupe sculpté du Prasat Kraham aura fourni le modèle des linteaux et des frontons des temples de Sūryavarman I ${ }^{\text {er }}$. Comme à Koh Ker, nous y retrouvons de façon distinctive la représentation de la déesse sous sa forme terrible (longtemps interprétée comme la dévote tamoule Kāraikkālammaiyār), assise au pied de Śiva dansant. Le motif se retrouve sensiblement à l'identique bien au-delà des fondations de la première moitié du $\mathrm{XI}^{\mathrm{e}}$ siècle, jusqu'au règne de Jayavarman VII 


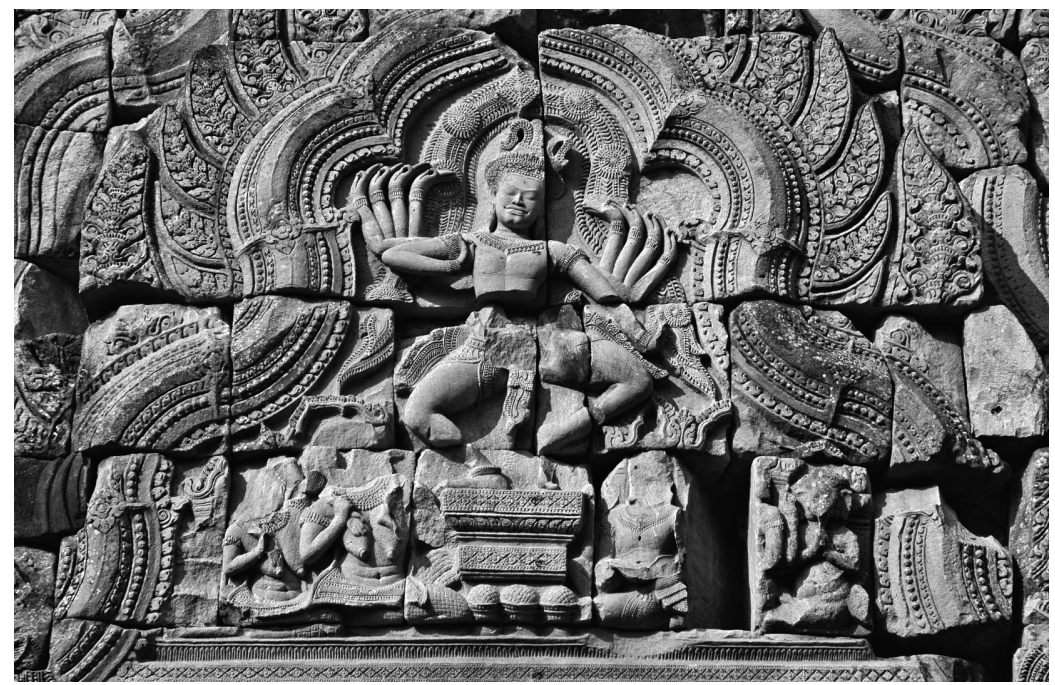

FIG. 15. - Śiva dansant et déesses tenant les membres de corps dépecés, fronton est de l'avant-corps de la tour centrale du temple de Phnom Rung (ph. auteur).

(à la suite de la Cāmuṇḍa du Prasat Thom, la déesse brandit alors tantôt une jambe ou un bras, tantôt une ou plusieurs têtes décapitées, fig. 15$)^{28}$.

Il y a peut-être là, pourra-t-on noter non sans malice, une certaine ironie de la recherche : la divinité, le Devarāja, dont on a certainement le plus recherché l'image en doutant même de son existence, se révèlerait être finalement celle pour laquelle fut réalisé l'un des groupes sculptés les plus grandioses et parmi les plus reproduits du Cambodge ancien.

\section{Bibliographie}

Assmann, Jan, 2010, Maât, L'Égypte pharaonique et l'idée de justice sociale, Paris, MdV éditeur.

BIARDEAU, Madeleine, 2002, Le Mahābhārata. Un récit fondateur du brahmanisme et son interprétation, 2 t., Paris, Seuil. 
BILlARD, Roger, 2006, « Dates des inscriptions du pays khmer» (travail revu et augmenté par J.C. Eade), Bulletin de l'École française d'Extrême-Orient 93, p. 395-428.

Bizot, François, 1989, Rāmaker ou L'amour symbolique de Rām et Setā, Recherches sur le bouddhisme khmer V, PEFEO CLV, Paris, EFEO.

BOURDONNEAU, Éric, 2011, « Nouvelles recherches sur Koh Ker (Chok Gargyar). Jayavarman IV et la maîtrise des mondes », Monuments et mémoires de la Fondation Eugène Piot 90, p. 93-141.

BRown, Peter, 2007 [1984], Le Culte des Saints. Son essor et sa fonction dans la chrétienté latine (trad. The Cult of the Saints. Its Rise and Function in Late Antiquity, 1981, Chicago, University of Chicago Press), Paris, Les Éditions du Cerf.

Chandler, David, 1996, Facing the Cambodian Past : Selected Essays 1971-1994, Chiang Mai, Silkworm Books.

CHENET, François, 2004, « Le secret de tous les secrets. Mort ou délivrance selon la Katha Upanishad », dans La mort et l'immortalité. Encyclopédie des savoirs et des croyances, F. Lenoir, J.-Ph. de Tonnac (dir.), Paris, Bayard, p. 580-590.

CEEDÈs, George, 1931, « Études cambodgiennes. XXVI - La date de Kòh Ker », Bulletin de l'École française d'Extrême-Orient 31, p. 12-18.

-, 1951, Inscriptions du Cambodge, vol. III, Collection de textes et documents sur l'Indochine III, Paris, École française d'ExtrêmeOrient.

-, 1952, Inscriptions du Cambodge, vol. IV, Collection de textes et documents sur l'Indochine III, Paris, École française d'Extrême-Orient.

—, 1961, « Les expressions vrah kamraten añ et kamraten jagat en vieuxkhmer », The Adyar Library Bulletin 25 (1-4), Jubilee volume, Madras, p. 447-460.

—, 1965, « Le rôle de la pyramide de Koh Ker dans l'évolution du templemontagne khmèr », Paranavitana Felicitation Volume, Colombo, 1965, p. 61-66.

CEEDÈS, George et Pierre DuPONT, 1943-1946, « Les stèles de Sdŏ̀k Kăk Thom, Phnom Sandak et Práh Vihãr », Bulletin de l'École française d'Extrême-Orient 43, p. 56-134.

DELAPORTE, Louis, 1880, Voyage au Cambodge. L'architecture Khmer, Paris, Librairie Ch. Delagrave.

ESTÈVE, Julia, 2009, Étude critique des phénomènes de syncrétisme religieux du Cambodge ancien, 2 vol., thèse de doctorat (9 juillet 2009), Paris, EPHE.

FiLliozAT, Jean, 1981, « Sur le çivaïsme et le bouddhisme du Cambodge, à propos de deux livres récents », Bulletin de l'École française d'Extrême-Orient 70, p. 59-100.

FILLIOZAT, Pierre-Sylvain, 2001, « Structures architecturales et iconographiques de temples de Śiva au Karnātaka (Sud de l'Inde) du $\mathrm{XI}^{\mathrm{e}}$ au 
XIII ${ }^{\mathrm{e}}$ siècle », Comptes rendus des séances de l'Académie des Inscriptions et Belles-Lettres, fascicule 1 (janvier-mars), p. 667-685.

—, 2007, « Note sur les modes de représentation de Sadāśiva dans l'ordonnance iconographique et architecturale du temple », dans Mélanges tantriques à la mémoire d'Hélène Brunner. Tantric Studies in Memory of Hélène Brunner, D. Goodall et A. Padoux (dir.), Collection Indologie $\mathrm{n}^{\mathrm{o}}$ 106, IFP/EFEO, p. 99-112.

GoudRIAAN, Teun, 1973, « Tumburu and His Sisters », Wiener Zeitschrift für die Kunde Südasiens 17, p. 49-95.

—, 1985, The Vinnāsikikhatantra : A Śaiva Tantra of the Left Current (édition et traduction), Delhi, Motilal Banarsidass.

JACQUES, Claude, 2009, « The opening phase of the Khmer Empire : the Koh Ker adventure », dans Jaya Koh Ker Project. Annual Report 2009, János Jelen (éd.), Budapest, Royal Angkor Foundation, p. 11-24.

KAIMAL, Padma, 1999, « Shiva Nataraja : Shifting Meanings of an Icon », The Art Bulletin 81, n³, p. 390-419.

LECLÈRE, Adhémard, 1917, Cambodge: fêtes civiles et religieuses, Annales du musée Guimet. Bibliothèque de vulgarisation, t. 42, Paris, Imprimerie nationale.

MALAMOUD, Charles, 1997, « Noirceur de l'écriture. Remarques sur un thème littéraire de l'Inde ancienne », dans Paroles à dire, paroles à écrire. Inde, Chine, Japon, V. Alleton (dir.), Paris, EHESS, p. 85-114.

—, 2002, Le Jumeau solaire, Paris, Seuil.

MALlmanN, Marie-Thérèse de, 1963, Les Enseignements iconographiques de l'Agni-Purana, Annales du musée Guimet, t. LXVII, Paris, P.U.F.

PADOUX, André, 2010, Comprendre le tantrisme. Les sources hindoues, Paris, Albin Michel.

PARMENTIER, Henri, 1939, L'Art khmèr classique. Monuments du quadrant Nord-Est, 2 vol., PEFEO XXIXbis, Paris, Les Éditions d'Art et d'Histoire.

PICHARD Pierre, L'Hernault Françoise, Boudignon Françoise et L. Thyagarajan L., 1994, Vingt ans après Tanjavur, Gangaikondacholapuram, Mémoires archéologiques 20, 2 vol., Paris, EFEO.

PorÉE-MASPÉRO, Èvelyne, 1962, Étude sur les rites agraires des Cambodgiens, t. 1, Paris, La Haye, Mouton \& Co.

Pou, Saveros, 2001, Nouvelles inscriptions du Cambodge II \& III (traduites et éditées), Collection de textes et documents sur l'Indochine XXII \& XXIII, Paris, EFEO.

SANDERSON, Alexis, 2003-2004, « The Śaiva Religion among the Khmers (part I) », Bulletin de l'École française d'Extrême-Orient 90-91, p. 349-462.

—, 2009, «The Śaiva Age : The Rise and Dominance of Śaivism during the Early Medieval Period », dans Genesis and Development of Tantrism, 
1382 COMPTES RENDUS DE L'ACADÉMIE DES INSCRIPTIONS

Shingo Einoo (éd.), Tokyo, University of Tokyo, Institute of Oriental Culture.

SchmitT, Jean-Claude, 1984, «La fabrique des saints», Annales. Economies, Sociétés, Civilisations 39, n², p. 286-300.

$*$

$* \quad *$

Le Président M. Michel ZinK, M. Franciscus Verellen et M. Azedine BeschaOUCH, associé étranger de l'Académie, interviennent après cette communication. 